INVESTIGATIONS OF NUCLEAR STRUCTURE AND NUCLEAR REACTIONS INDUCED BY COMPLEX PROJECTILES

Principal Investigator: D. G. Sarantites

Department of Chemistry

Washington University, St. Louis, MO 63130

$\underbrace{2}$

- $\cdots \cdots$

Technical Progress Report (COO-4052-16)

submitted to the

Department of Energy

under contract EY-76-S-02-4052-AOO

for the period

November 1, 1977 through October 31, 1978

This report was prepared as an account of work sponsored by the United States Government. Neither the United States nor the United States Department of Energy, nor any of their employees, nor any of their contractors, subcontractors, or their employees, makes any warranty, express or implied, or assumes any legal liability or responsibility for the accuracy, comple teness or usefulness of any information, apparatus, product of process disclosed, or represents that its use would not infringe privately owned rights. 


\section{DISCLAIMER}

This report was prepared as an account of work sponsored by an agency of the United States Government. Neither the United States Government nor any agency Thereof, nor any of their employees, makes any warranty, express or implied, or assumes any legal liability or responsibility for the accuracy, completeness, or usefulness of any information, apparatus, product, or process disclosed, or represents that its use would not infringe privately owned rights. Reference herein to any specific commercial product, process, or service by trade name, trademark, manufacturer, or otherwise does not necessarily constitute or imply its endorsement, recommendation, or favoring by the United States Government or any agency thereof. The views and opinions of authors expressed herein do not necessarily state or reflect those of the United States Government or any agency thereof. 


\section{DISCLAIMER}

Portions of this document may be illegible in electronic image products. Images are produced from the best available original document. 
TABLE OF CONTENTS

Part 1. PRESENT STATUS OF THE RESEARCH PROGRAM

1.1 FUSION REACTIONS . . . . . . . . . . . . . . . . . 1

a) "Gamma-Ray Multiplicities in Evaporation Residues Formed in Bombardments of $150_{\mathrm{Nd}}$ by ${ }^{20} \mathrm{Ne}^{\prime \prime}(\mathrm{COO}-4052-02) \ldots . . . . .1$

b) " $\gamma$-Ray Multiplicities from Nuclear Reactions: Theory, Instrumentation and Analysis" (COO-4052-03) . . ....... 2

c) "Reactions of ${ }^{20} \mathrm{Ne}$ on ${ }^{150} \mathrm{Nd}$ at $175 \mathrm{MeV}^{\prime}(\mathrm{COO}-4052-04)$.... 2

d) "Multiplicity of the $\gamma$-cascades in the ${ }^{61} \mathrm{Cu}$ Continuum: Dependence on Excitation Energy of the Entry. States: (CO0-4052-05) .................... 3

e). "Angular Momentum Effects in Pre-Equilibrium Evaporation Processes" (C00-4052-06)................ 4

f) "Pre-Equilibrium Effects in Fusion of ${ }^{12} \mathrm{C}$ and ${ }^{158} \mathrm{Gd}$ " $(\mathrm{c} 00-4052-11)$. . . . . . . . . . . . . . 5

g) "Pre-Equilibrium Particle Emission from Fusion of $12 \mathrm{C}+158 \mathrm{Gd}$ and $20 \mathrm{Ne}+150 \mathrm{Nd}(\mathrm{COO}-4052-12)$. . . . . . 7

h) "Multipolarities of the Yrast and Statistical Cascades

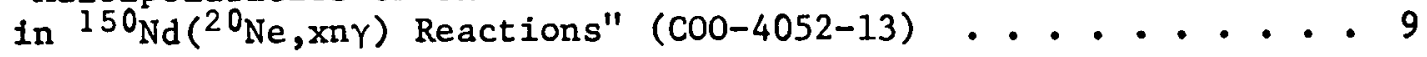

1) "Dependence of $\gamma$-Multiplicity on Transition Energy of the Continuum from ${ }^{20} \mathrm{Ne}+{ }^{150} \mathrm{Nd}$ Reactions .......... 10

j) "Gross Structure of the Spectrum of the Yrast and the Statistical Region" . . . . . . . . . . . . . . 13

k) "Evidence for Pre-Equilibrium Effects in $160+154 \mathrm{sm}$ Fusion Reactions................... 17

1) "Gamma-Ray Multiplicities from Reactions Forming the Compound Nucleus $100_{\mathrm{Ru}}$. . . . . . . . . . . . . . 21

m) "Multiplicities of $\gamma$-transitions Observed in ${ }^{6} \mathrm{Li}$ Induced Reactions" . . . . . . . . . . . . . 24

n) "Gamma-ray Multiplicities in Reaction Products from 80 MeV Proton Bombardments of $60 \mathrm{Ni}$ and ${ }^{169}{ }^{\mathrm{Tm}}{ }^{\prime \prime}$. . . . . . . 25

1.2 STRONGLY DAMPED HEAVY-ION COLLISIONS . . . . . . . . . . . 26

a) "Angular Momentum Transfer in Deep Inelastic Scattering" . . . 26

b) Energy Dissipation by Charged Particle Emission in Deep Inelastic Scattering" . . . ........... 56 
1.3 NUCLEAR STRUCTURE AT HIGH ANGULAR MOMENTUM . . . . • . • • . . . 57

a) "Identification of a $\mathrm{J} \simeq 32$ Isomeric Yrast-trap in ${ }^{148} \mathrm{~Tb} "$. . 58 (C00-4052-14)

b) "Discrete High-spin States in the Yrast Sequence of $164 \mathrm{Yb} "$. . 59

c) "High-spin States in ${ }^{132} \mathrm{Ce}^{1}$.................... 62

d) "Lifetimes of Ground-band States in ${ }^{192} \mathrm{Pt}$ and ${ }^{194} \mathrm{Pt}$ " . . . 66 (C00-4052-10)

e) "Structure of the Weakly Deformed ${ }^{97} \mathrm{Ru}$ and ${ }^{99} \mathrm{Ru}$ Nuclei" . . . 67 (C00-4052-15)

Part 2. PRESENT STATUS OF INSTRUMENTATION AND ANALYSIS

2.1 APPARATUS FOR $\gamma$-RAY AND PARTICLE MULTIPLICITY EXPERIMENTS • • • • 69

a) "Anti-Compton Spectrometer". . . . . . . . . . . . 69

b) "'Urchin". Shields" . . . . . . . . . . . . . . 69

c) Two 30-cm Diameter Multi-coincidence Scattering Chambers" . : 70

d) "A 50-cm Diameter Multiple-coincidence Scattering Chamber" . . 70

e) "Multiplicity Routing Box" . . . . . . . . . . . . 71

2.2 A MAGNETIC LENS-Si(Li) VERSATILE CONVERSION ELECTRON SPECTROMETER • 71

a) "Conversion Coefficients from In-beam Heavy-ion Reactions" • . 74

b) "Conversion Electrons from the Continuum" ......... 79

c). "Multiplicity. Experiments with Conversion Electrons" . . . . 79

2.3 A TOTAL ENERGY OR COINCIDENCE-SUMMING SPECTROMETER . . . . • . . . 84

2.4 DETECTION EQUIPMENT . . . . . . . . . . . . . . . . . . . . . . . . . . . .

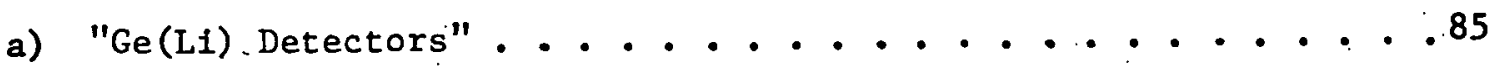
b) "Light-particle $\Delta \mathrm{ExE}^{\prime}$. . . . . . . . . . . . . 85
c) "Heavy-ion $\triangle$ ExE Telescopes" ...................... 85
d) "Neutron Detectors" . . . . . . . . . . . . 85
e) "Si(Li)-Conversion Electron Detectors" . . . . . . . 86
f) "Large NaI Detectors" ................ . . . . . . 66 
2.5 DATA ACQUISITION AND REDUCTION . . . . . . . . . . 86
a) "The ORIC SEL-computers as Data Acquisition Systems for Experiments in this Project" . . . . . . . . . . . 86
b) "Data Reduction at the ORIC" . . . . . . . . . . 87
c) "Data Reduction at Washington University" . . . . . . . . 87

Part 3. SCIENTIFIC AND TEChNICAL PERSONNEL . . . . . . . . . . . 89

Part 4. PUBLICATIONS ................... 89

4.1 Papers published in 1976-1978 . . . . . . . . . 89

4.2 Papers submitted for publication . . . . . . . . . 90

4.3 Presentations at Professional Meetings . . . . . . . . 91 
PART 1. PRESENT STATUS OF THE RESEARCH PROGRAM

In this section work in progress or completed during the last two contract

years will be briefly summarized. The affiliation of collaborators is indi-

- cated by appropriate footnotes.

\title{
1.1 FUSION REACTIONS
}

PHYSICALREVIEWC

VOLUME 14; NUMBER6, P. 2138

DECEMBER 1976

a)

$$
\begin{aligned}
& \text { Gamma-ray multiplicities in evaporation residues formed in bombardments } \\
& \text { of }{ }^{150} \mathrm{Nd} \text { by }{ }^{20} \mathrm{Ne}+
\end{aligned}
$$

D. G. Sarantites

Department of Chemistry, Washington University, St. Louis, Missouri 63130

J. H. Barker

Department of Physics, St. Louis University, St. Louis, Missouri 63103

M. L. Halbert, D. C. Hensley, R. A. Dayras, E. Eichler, and N. R. Johnson

Oak Ridge National Laboratory. Oak Ridge, Tennessee 37830

\author{
S. A. Gronemeyer \\ Department of Physics, Washington University. St. Louis, Missouri 63130
}

(Received 28 June 1976)

The first three moments of the multiplicity distribution were deduced for the $\gamma$-ray cascades in the evaporation residues produced in bombardments of ${ }^{150} \mathrm{Nd}$ with ${ }^{30} \mathrm{Ne}$ at four energies from 127.7 to $172.4 \mathrm{MeV}$ by means of measurements of the first and higher order coincidence rates between a $\mathrm{Ge}(\mathrm{Li})$ spectrometer and eight $\mathrm{NaI}(\mathrm{TI})$ detectors. The cross sections for the production of ${ }^{160-165} \mathrm{Yb}$ and ${ }^{136-161} \mathrm{Er}$ were also measured. The average multiplicity $\left\langle M_{j}\right\rangle$ and the width of the multiplicity distribution $\sigma_{M_{J}}$ for cascades via a state of spin $J$ were found to be independent of $J$. For. a given product, $(M)$ and $\sigma_{M}$ increase with bombardment energy but for fixed energy they decrease with increasing number of emitted neutrons. The observed values of $\langle M\rangle$ and $\sigma_{M}$ range from 11 to 32 and 6 to 9 , respectively. The multiplicities $\langle M\rangle$ in the $\left({ }^{20} \mathrm{Ne}, x n a\right)$ products are considerably lower than those for $\left({ }^{20} \mathrm{Ne}, x n\right)$ but the widths $\sigma_{M}$ are the same for a given value of $x$. The skewness of the multiplicity distribution is negative, approaching zero as the number of emitted neutrons increases. Angular momentum distributions were deduced (a) for each product prior to $\gamma$ decay and (b) for the initial compound nuclei which lead to each product. In each case these were found to overlap extensively for several neighboring neutron numbers. The $J$ distribution in ${ }^{163} \mathrm{Yb}$ extends up to $J \simeq 85$ prior to $\gamma$ decay. At the higher energies a emission plays an important role in the deexcitation process by removing on the average an angular momentum of $-10 \pi$. The present results suggest that $\alpha$ emission follows neutron evaporation. The $J$ distributions obtained are compared with the predictions of the Bass model for fusing collisions. For the lower energies excellent agreement is observed. For the higher energies the Bass model prediction exceeds the measured $x n$ and $x n a$ cross sections at all $J$, indicating that other exit channels account for an appreciable fraction of the fusion cross section.

[NUCLEAR REACTIONS ${ }^{150} \mathrm{Nd}\left({ }^{20} \mathrm{Ne}, x m \gamma\right),{ }^{150} \mathrm{Nd}\left({ }^{20} \mathrm{Ne}, x n \alpha \gamma\right), x=5-10, E=127.7-$ $172.4 \mathrm{MeV}$; measured $\sigma\left(E, E_{\gamma}\right), \gamma$-ray multiplicities; deduced $J$ distributions in evaporation residues. Enriched target.

Reprints of this article were submitted last year (c00-4052-02). 
NUCLEAR INSTRUMENTS AND METHODS $145(1977) 295-310$ : C NORTH-HOLLAND PUBLISHING CO.

\title{
b) $\gamma$-RAY MULTIPLICITIES FROM NUCLEAR REACTIONS: THEORY, INSTRUMENTATION AND ANALYSIS
}

\author{
L. WESTERBERG, D. G. SARANTITES, R. LOVETT, J. T. HOOD
}

Department of Chemistry, Washington Liniversity. St. Louis, Missouri 63130, U.S.A.

\section{J. H. BARKER}

Department of Physics. St. Louis University. St. Louis, Missouri 63103, U.S.A.

C. M. CURRIE and N. MULLANI

Radiation Sciences, Washingion University, St. Louis, Missouri 63130, U.S.A.

Received 23 March 1977

Expressions for interpreting $\gamma$-ray multiplicity measurements with a $\mathrm{Ge}(\mathrm{Li})$ and $N \mathrm{NaI}$ detectors are derived. A scattering chamber designed to allow measurements of multiple coincidences between a $\mathrm{Ge}(\mathrm{Li})$ detector and/or particle detectors and up to $12 \mathrm{Nal}$ detectors is described. A special multiplicity recording unit has been constructed. It employs the strobed overlap coincidence technique and it is suitable for routing a multichannel analyzer or for interfacing to a computer. Details of the analysis procedure are discussed. The application of a computer code to multiplicity calculations is described.

Reprints of this article are appended (CO0-4052-03).

PHYSICAL REVIEW C

VOLUME 17, NUMBER I, P. 155

JANUARY 1978

c)

\author{
Reactions of ${ }^{20} \mathrm{Ne}$ on ${ }^{150} \mathrm{Nd}$ at $175 \mathrm{MeV}^{*}$ \\ M. L. Halbert, R. A. Dayras, R. L. Ferguson, and F. Plasil \\ Oak Ridge National Laboratory, $\dagger$ Oak Ridge, Tennessee 37830 \\ D. G. Sarantites \\ Department of Chemistry. Washington University, St. Louis, Missouri 63130
} (Received 29 July 1977)

Energy spectra of charged-particle reaction products from bombardment of ${ }^{150} \mathrm{Nd}$ by $175-$ $\mathrm{MeV}{ }^{20} \mathrm{Ne}$ were measured with three $\Delta E, E$ telescopes at angles from $3.5^{\circ}$ to $100^{\circ}$. The evaporation residues and symmetric-fission fragments were easily identified; their total cross sections were $1150 \pm 90$ and $280 \pm 35 \mathrm{mb}$, respectively. The sum of these numbers is in good agreement with the fusion cross section predicted by the Bass model. The elastic cross section drops to $\frac{1}{4}$. of the Rutherford cross section at $30.4 \pm 0.2^{\circ}$ (lab), which implies that the total region cross section is $2.63 \mathrm{~b}$. Products from quasielastic and from strongly damped collisions were observed; their angle-integrated yields account for the balance of the reaction cross section.

NUCLEAR REACTIONS ${ }^{150} \mathrm{Nd}\left({ }^{20} \mathrm{Ne},{ }^{20} \mathrm{Ne}\right),\left({ }^{20} \mathrm{Ne}, F\right),\left({ }^{20} \mathrm{Ne}, X\right), E=175.3 \mathrm{MeV}$; measured elastic, fission, fusion, evaporation residue $\sigma(\theta)$; deduced total reacaction $\sigma$.

Reprints of this article are appended (Report No. CO0-4052-4): 


\title{
Multiplicity of the $\gamma$ cascades in the ${ }^{61} \mathrm{Cu}$ continuum*: Dependence on excitation energy of the entry states
}

\author{
J. H. Barker' \\ Department of Physics, Saint Louis, University. St. Louis, Missouri 63103 \\ D. G. Sarantites \\ Department of Chemistry. Washington University. St. Louis, Missouri 63130 \\ Ö. Skeppstedt, E. Wallander, G. Alenius, and S. -E. Amell \\ Depariment of Physics. Chalmers University of Technology, Gothenburg. Sweden \\ (Received 9 June 1977)
}

The multiplicity of the $\gamma$ cascades in the ${ }^{61} \mathrm{Cu}$ continuum entering 17 levels populated via the ${ }^{38} \mathrm{Ni}\left({ }^{4} \mathrm{He}, p \gamma\right)^{61} \mathrm{Cu}$ reaction have been measured for incident ${ }^{4} \mathrm{He}$ energies of 17.0 and $19.5 \mathrm{MeV}$. A general trend of increasing multiplicity of side feeding with increasing excitation energy of the entry states is established. For excitations between 6.0 and $12.5 \mathrm{MeV}$ in ${ }^{61} \mathrm{Cu}$ the side-feeding multiplicities vary between 1.2 and 4.0 , while the total multiplicities vary between 4.0 and 6.8 . The side-feeding mufilplicities from the $19.5-\mathrm{MeV}$ experiment are about 0.7 units higher than those from the $17.0-\mathrm{MeV}$ experiment. Detailed statistical model calculations which include explicitly. $\gamma$-ray competition reproduce the trends and the magnitudes of the measured multiplicities.

PACS numbers: $25.60 . \mathrm{Fb}, 27.50+\mathrm{e}, 46.0+. \mathrm{m}$

[NUCLEAR REACTIONS ${ }^{58} \mathrm{Ni}(\alpha, p \gamma), 17.0$ and $19.5 \mathrm{MeV}$; measured $\gamma$-ray multi-] plicites v.S $E^{*}$; compound statistical model analysis.<smiles>[14CH3]</smiles>

.

Preprint of this article was submitted last year (Report No. C00-4052-05).

A xerox copy of the proof is appended. 
PHYSICAL REVIEW C

VOLUME 1 7, NUMBER 2, P. 601

FEBR UARY 1978

e)

Angular-momentum effects in pre-equilibrium processes

\author{
D. G. Sarantites and L. Westerberg \\ Department of Chemistry. Washington University, "St. Louis, Missouri 63130
}

R. A. Dayras, M. L. Halbert, and D. C. Hensley

Oak Ridge National Laboratory, † Oak Ridge. Tennessee 37830

J. H. Barker

Department of Physics, Saint Louis University. St. Louis, Missouri 63103

(Received 8 July 1977)

Cross sections and the first three moments of the $\gamma$-ray multiplicity distribution were measured for many transitions corresponding to $x n$ and $x n \alpha$ products from bombardments of ${ }^{166} \mathrm{Er}$ with ${ }^{4} \mathrm{He},{ }^{150} \mathrm{Nd}$ with ${ }^{20} \mathrm{Ne}$, and ${ }^{130} \mathrm{Te}$ with ${ }^{40} \mathrm{Ar}$. The ${ }^{4} \mathrm{He}$ and ${ }^{20} \mathrm{Ne}$ bombarding energies were chosen to produce ${ }^{170} \mathrm{Yb}$ compound nuclei at similar excitation energies, approximately 63, 77, and $92 \mathrm{MeV}$. A Ge(Li)-NaI anti-Compton spectrometer was used in coincidence with nine $5.1-\mathrm{cm}$ by $7.6 \mathrm{~cm} \mathrm{NaI}(\mathrm{Tl})$ detectors. For the reactions $\left({ }^{20} \mathrm{Ne}, x n\right)$ and $\left({ }^{40} \mathrm{Ar}, x n\right)$, the average $\gamma$-ray multiplicity $\langle M\rangle_{x}$ increases linearly with decreasing $x$ as expected from the statistical decay of compound nuclei. In the $\left({ }^{4} \mathrm{He}, x n\right)$ reactions, $\langle M\rangle_{x}$ is similar for large $x$ but for small $x$ it decreases substantially with decreasing $x$. The latter corresponds to a shift in the population toward the low $J$ values of levels in ${ }^{170-x} \mathrm{Yb}$ prior to $\gamma$ decay. Also, in the few-neutron channels the average multiplicity associated with a given gating transition in the residual nucleus is much smaller for transitions between lowlying levels. These features, absent in the heavy-ion bombardments, indicate a strong contribution from preequilibrium neutron emission. The measured $\left({ }^{4} \mathrm{He}, x n\right)$ cross sections are in reasonable agreement with those calculated from the geometry-dependent hybrid model of pre-equilibrium emission except for the smallest values of $x$; the latter results and the low $\langle M\rangle_{x}$ values for $x \leq 4$ suggest that more than one preequilibrium neutron is emitted in these channels.

[NUCLEAR REACTIONS $\left.{ }^{150} \mathrm{Nd}\left({ }^{20} \mathrm{Ne}, x n \gamma\right),\left({ }^{20} \mathrm{Ne}, x n \alpha \gamma\right), x=4-8, E=94.2,110.7\right]$ $\mathrm{MeV} ;{ }^{130} \mathrm{Te}\left({ }^{40} \mathrm{Ar}, x n \gamma\right), x=3-5, E=154.5 \mathrm{MeV} ;{ }^{166} \mathrm{Er}\left({ }^{4} \mathrm{He}, x n \gamma\right),\left({ }^{4} \mathrm{He}, x n \alpha \gamma\right), x$ $=2-8 ; E=67.6-95.3 \mathrm{MeV}$; measured $\sigma(E, E \gamma), \gamma$-ray multiplicities; deduced $J$ distributions in evaporation residues. Enriched target, anti-Compton $\mathrm{Ge}(\mathrm{Li})$ -

NaI spectrometer.

Reprints of this article are appended (c00-4052-06) 
f)

PRE-EQUILIBRIUM EFFECTS IN FUSION OF ${ }^{12} \mathrm{C}$ AND ${ }^{158} \mathrm{Gd}$

\begin{abstract}
D. G. Sarantites and L. Westerberg*
Department of Chemistry, Washington University

St. Louis, Missouri 63130
\end{abstract}

M. L. Halbert, R. A. Dayras, and D. C. Hensley

Oak Ridge National Laboratory

Oak Ridge, Tennessee 37830

J. H. Barker

Department of Physics, St. Louis University

St. Louis, Missouri 63103

\title{
ABSTRACT
}

Cross sections and the first three moments of the $\gamma$-ray multiplicity distribution were measured for many transitions in a series of $170-x_{Y b}$ and $166-x_{E r}$ products from bombardments of ${ }^{158} \mathrm{Gd}$ with ${ }^{12} \mathrm{C}$ at five energies corresponding to excitation in ${ }^{170} \mathrm{Yb}$ between 94 and $143 \mathrm{MeV}$. The average multiplicity for each xn channel increases with energy at first and then levels off at $\sim 21$. For the $\alpha \times n$ channels saturation values between 16 and 20 are found. The excitation functions for $166-x_{E r}$ indicate that the isotopes with small $x$ are formed primarily by $2 p(x+2) n$ evaporation and the ones with large $x$ by $\alpha x n$ emission. A corresponding discontinuity in the average. multiplicity vs. $x$ is observed. These features are absent in $20 \mathrm{Ne}+150 \mathrm{Nd}$ reactions at the same 
ar: $\because \cdots$

170 Yb excitation, but are present in the ${ }^{166} \mathrm{Er}\left({ }^{4} \mathrm{He}, \mathrm{xn}\right)$ reac-

tions at excitations from 63 to $92 \mathrm{MeV}$. The present results are clear indications of pre-equilibrium emission of neutrons and possibly of $\alpha$-particles, setting in at bombarding energies $\sim 10 \mathrm{MeV} / \mathrm{amu}$.

Zerox of title and abstract from preprint by D. G. Sarantites et al.

This work has been accepted for publication in the Physical Review in April, 1978. A preprint is appended (see Report No. C00-4052-09). 
g)

PRE-EQUILIBRIUM PARTICLE EMISSION

FROM FUSION OF ${ }^{12} \mathrm{C}+{ }^{158}$ Gd AND ${ }^{20} \mathrm{Ne}+{ }^{150} \mathrm{Nd}$

L. Westerberg ${ }^{*}$ and D. G. Sarantites

Department of Chemistry, Washington University

St. Louis, Missouri 63130

D. C. Hensley, R. A. Dayras and M. L. Halbert Oak Ridge National Laboratory

Oak Ridge, Tennessee 37830

\section{J. H. Barker}

Department of Physics, St. Louis University

St. Louis, Missouri 63130

\section{ABSTRACT}

Neutron time-of-flight spectra and $\alpha$-particle spectra were obtained in coincidence with $\gamma$-rays characteristic of specific evaporation residues in the reactions $174.8 \mathrm{MeV} 20_{\mathrm{Ne}}+{ }^{150} \mathrm{Nd}$ and $152.0 \mathrm{MeV}$ ${ }^{12} \mathrm{C}+{ }^{158} \mathrm{Gd}$, producing ${ }^{170} \mathrm{Yb}$ at 134.2 and $132.0 \mathrm{MeV}$ of excitation. The average multiplicity of $\gamma$-rays associated with such coincident events were also determined. The neutron spectra from the ${ }^{20} \mathrm{Ne}$ reactions are characteristic of equilibrium evaporation processes. They show a temperature $t \sim 2 \mathrm{MeV}$, in agreement with a prediction from level-density parameters based on data at far lower excitation energy. The neutron spectra from the ${ }^{12} \mathrm{C}$ reactions show a lowtemperature equilibrium component (average $t \sim 1.8 \mathrm{MeV}$ ) and a hightemperature ( $\sim 2 \mathrm{MeV}$ ) pre-equilibrium component. The latter observation may be interpreted as evidence for the transient existence of 
a hot spot involving no more than 25 nucleons. The estimated number of pre-equilibrium neutrons emitted varies from 1.8 to 0.6 for the 8 n to $10 \mathrm{n}$ products and from 1.2 to 0.25 for the $\alpha 6 n$ to $\alpha 9 n$ products. The fraction of $166-x$ Er products formed by $2 p(x+2) n$ rather than $\alpha x n$ emission decreases with increasing $x$ for both the ${ }^{20} \mathrm{Ne}$ and ${ }^{12} \mathrm{C}$ reactions, and is roughly twice as large for ${ }^{12} \mathrm{C}$. The high-energy portions of the $\alpha$-particle spectra from $\left({ }^{12} C, \alpha \times n\right)$ for small $x$ exhibit a strono dependence on exit channel and on angle of emission, indicating clearly that these alphas are emitted from a non-equilibrated system. The angular correlations of the higher-energy $\alpha$-particles from $\left({ }^{12} c, \alpha 7 n\right)$ to $\left({ }^{12} \mathrm{C}, \alpha 10 \mathrm{n}\right)$ all have the same shape, suggesting that in these channels the a-emission occurs first. The pre-equilibrium emission accounts for $(16 \pm 7) \%$ and $(28 \pm 6) \%$ of the total $\left({ }^{12} \mathrm{C}, \times n\right)$ and $\left({ }^{12} \mathrm{C}, \alpha \times n\right)$ cross sections, respectively. For these reactions the average multiplicities $\langle M\rangle_{x n}$ and $\langle M\rangle_{\alpha \times n}$ from coincidences with neutrons and $\alpha$-particles of ail energies saturate at $\sim 20.5$ for $x \leq 9$ and $\leq 8$, respectively, showing that the pre-equilibrium emission limits the angular momentum that can be imparted to the product nuclei prior to $\gamma$-decay. For $\left({ }^{20} \mathrm{Ne}, \alpha \times n\right)$ evidence for such a limiting effect was found for $x \leq 7$ and the $\alpha$-particle spectra show some hardening for $x \leq 7$, but there is no evidence for pre-equilibirum emission in the $\left({ }^{20} \mathrm{Ne}, x_{n}\right)$ reactions. The multiplicity from $\left({ }^{12} \mathrm{C}, \alpha 8 n\right)$ associated with $E_{\alpha} \geq 24 \mathrm{MeV}$ was found to be lower at forward $\alpha$-emission angles, apparently another manifestation of pre-equilibrium emission.

This work has been accepted for publication in "The Physical Review" in April 1978. A preprint is appended (See Report No. C00-4052-12) 
h)

$$
\begin{aligned}
& \text { MULTIPOLARITIES OF THE YRAST AND STATISTICAL CASCADES } \\
& \text { IN }{ }^{150} \mathrm{Nd}\left({ }^{20} \mathrm{Ne}, \mathrm{XnY}\right) \text { REACTIONS } \\
& \text { L. Westerberg, D. G. Sarantites, and K. Geoffroy } \\
& \text { Department of Chemistry, Washington University, } \\
& \text { St. Louis, Missouri } 63130
\end{aligned}
$$

R. A. Dayras, J. R. Beene, M. L. Halbert and D. C. Hensley

Oak Ridge National Laboratory, Oak Ridge, Tennessee 37830

\author{
J. H. Barker \\ Department of Physics, St. Louis. University, \\ St. Louis, Missouri 63103
}

The total conversion coefficient, $\alpha$, of the $\gamma$-ray continum has been determined as a function of $\gamma$-ray energy from 0.5 to $2.5 \mathrm{MeV}$. The results are consistent with a mixture of $E 1$ and $E 2$ for $E_{\gamma} \geq 1.0 \mathrm{MeV}$, with a smooth increase of the El fraction with $E_{\gamma}$ up to $\sim 1.5 \mathrm{MeV}$. At lower energy, in the upper half of the yrast region, $\alpha$ shows the expected dominance of E2 radiation, but for the lower half an increasing $M I$ contribution is found.

Xerox of the title and abstraction from preprint.

This work has been accepted for publication in "physical Review Letters." A preprint is appended (See Report No. COO-4052-13). 
(1) "DEPENDENCE OF $\gamma$-MULTIPLICITY ON TRANSITION ENERGY OF THE CONTINUUM FROM ${ }^{20} \mathrm{Ne}+{ }^{150}{ }_{\text {Nen }}^{d}$ REACTIONS

D. G. Sarantites, L. Westerberg, K. Geoffroy, M. L. Halbert, ${ }^{*}$ R. A. Dayras, * J. R. Benne, ${ }^{*}$ D. C. Hensley ${ }^{*}$ and J. H. Barker ${ }^{+}$

Useful information about the properties of nuclei at high spin can be obtained from the study of the $\gamma$-ray multiplicity as a function of transition energy of the continuum. In this way the effects of the entry line where particle decay is replaced by the beginning of the $\gamma$-cascades as well as the nature and position of the yrast line can effectively be studied.

Difficulties with such measurements are twofold. Firstly, when a NaI is employed as the gating detector, the presence of Compton events from higher energy transitions into the gate presents some difficulty in such measurements, because unfolding of coincidence spectra from coincidences of all orders (all folds) are required. Secondly, the 0 -fold $\gamma$-spectra are contaminated considerably with background radiation and are thus not useful.

We have bypassed these difficulties by employing the magnetic-lens Si(L1) electron spectrometer as the gating detector. This detector is better shielded from extraneous radiation and has an essentially $\delta$-function response. The multiplicity was derived from the distribution of coincidences with $0-, 1-, 2-, \ldots, 7 \mathrm{NaI}$ detectors with conversion electrons between $400-2500 \mathrm{keV}$ following the ${ }^{20} \mathrm{Ne}+{ }^{150} \mathrm{Nd}$ reactions of 115 and 130 $\mathrm{MeV}$.

The obtained multiplicities are shown in Fig. 1. For the $130 \mathrm{MeV}$

\footnotetext{
*Physics Division, ORNL.

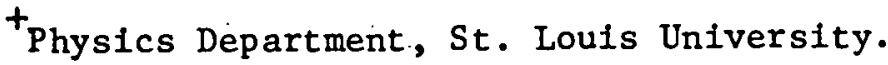


Fir. 1. Dependence of $\gamma$-ray multiplicity as a function of the transition energy of the continuum from ${ }^{20} \mathrm{Ne}+{ }^{150} \mathrm{Nd}$ reactions at 115 and $130 \mathrm{MeV}$. 


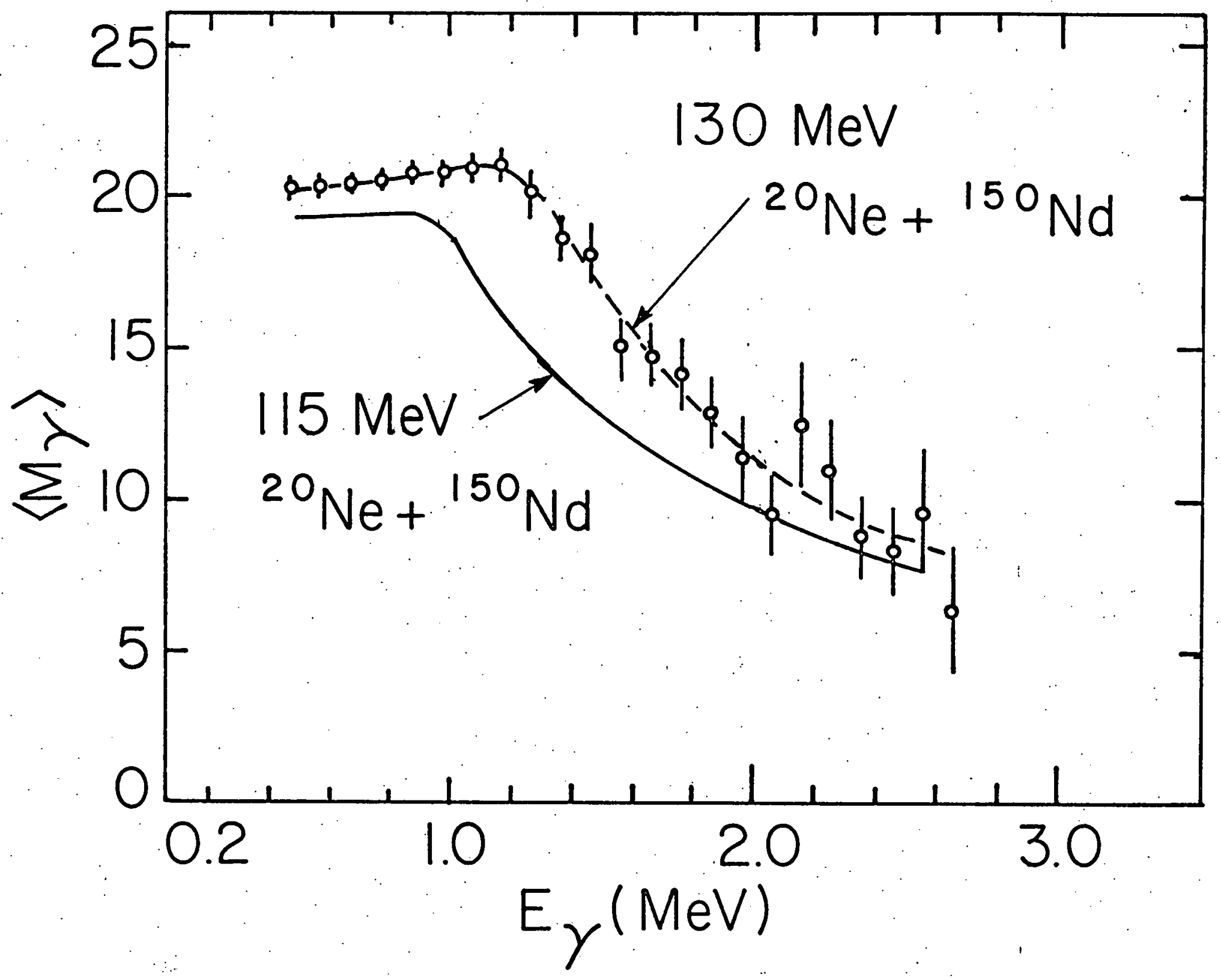

Fig. I 
experiment the multiplicity is increasing slowly with transition energy up to the midpoint of the end of the yrast bump (See Fig. 1 in C00-4052-13), and then decreases to about half the value when the statistical region is reached. This drop of the multiplicity with transition energy follows the end of the yrast region which correlates well with the amount of angular momentum deposited in the system. The latter is easily varied by changing the bombardment energy.

A continued decrease in $\left\langle\mathrm{M}_{\gamma}\right\rangle$ with transition energy was found for $\mathrm{E}_{\gamma}$ up to $\sim 5 \mathrm{MeV}$. These observations can be explained by assuming that the entry state line is not parallel to the yrast line but it is further removed from it at lower $\mathrm{J}$ values.

A model for a quantitative analysis of this effect taking into account a statistical decay with adjustable strength for the lower multipoles is now being developed to explain these data.

(j). GROSS STRUCTURE OF THE SPECTRUM OF THE YRAST AND THE STATISTICAL REGION

D. G. Sarantites, L. Westerberg, K. Geoffroy, J. H. Barker, M. L. Halbert, R. A. Dayras, J. R. Beene and D. C. Hensley

The spectrum of the electromagnetic radiation from heavy-ion induced reactions exhibits two main components. ${ }^{1,2}$. The lower energy part which is characterized by a rather steep edge at its high energy side is believed to be associated with the yrast cascades and it was recently shown ${ }^{3}$ to

$1_{\text {J.0. New.ton et }}$ al., Phys. Rev. Letters 34, 99 (1974).

2M. V. Banaschick, et al., Phys. Rev. Letters 34, 892 (1975).

${ }^{3} \mathrm{~L}$. Westerberg et al., (Appended preprint c00-4052-11). 
consist of M1 transitions in 1ts lowest part and E2 transitions in its upper energy part. The higher energy part of the $\gamma$-spectrum, which appears to decrease exponentially is believed to arise from the statistical cascades that proceed toward the yrast line. The gross structure of the yrast region reflects the dependence of the moment-of-inertia of the nucleus as a function of angular momentum and therefore energy via the usual relation $E_{\text {tot }}=\frac{\hbar^{2}}{2 I} \mathrm{~J}(\mathrm{~J}+1)$. On the other hand the shape of the spectrum in the statistical region reflects the dependence of the density of state at high angular momentum in the region of $i 8 \mathrm{MeV}$ above the yrast line. Predictions of an $E_{n} \cdot e^{-E_{n} / t}$ with $n=2-4$ have been recently made by Liotta and Sorensen ${ }^{4}$ for the energy dependence in the statistical region on the basis of a microscopic calculation.

We have investigated the gross structure of both the yrast and the statistical region by means of the ${ }^{20} \mathrm{Ne}+{ }^{150} \mathrm{Nd}$ reactions at 115 and 130 $\mathrm{MeV}$ where the $6 \mathrm{n}$ and $7 \mathrm{n}$ channels have their maxima and account for $260 \%$ of the fusion cross section. We have unfolded spectra obtained in a $2^{\prime \prime}$ diameter 3" long NaI detector in coincidence with 1 or more, 2 or more, etc. detectors of a multiplicity array. The results from the $130 \mathrm{MeV}$ bombardment are shown in Fig. 2. A shift of the yrast bump to high energy is clearly seen as high fold concidences are required. For example, in the $(6+7)$-fold spectrum the low multiplicity $\left({ }^{20} \mathrm{Ne}, 7 n\right)$ as well as the low multiplicity cascades that enter midstream the yrast cascade sequence are suppressed considerably. Therefore, the spectrum is indicative of what might be expected if the yrast cascade is entered at the top. Efforts are being made at the present to extract the moment-of-inertia as a

${ }^{4}$ R.J. Liotta and R.A. Sorensen, Nuc1. Phys. A297; 1.36. (1978). 
Fig 2. Spectra of the $\gamma$-rays from the ${ }^{20} \mathrm{Ne}+{ }^{150} \mathrm{Nd}$ reactions at $115 \mathrm{MeV}$ observed with a $2^{\prime \prime} \times 3^{\prime \prime} \mathrm{NaI}$ detector (the unfolded spectra are shown) in coincidence with $0-7,1-7,2-7, \ldots ., 6-7$ detectors of the 7. detector multiplicity array. 


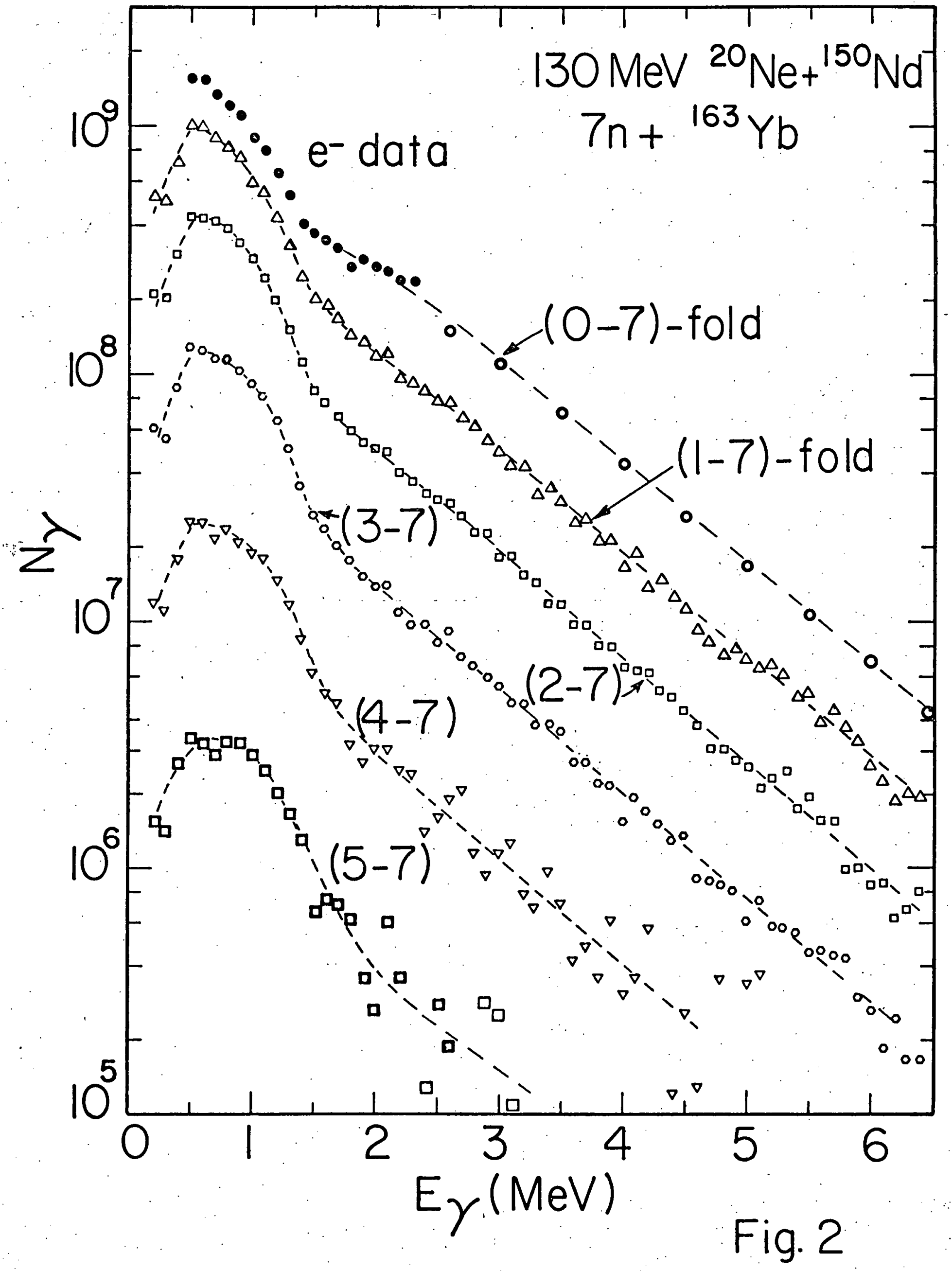


function of angular momentum (up to $240 \pi$ ) from such spectra.

The shape of the spectrum in the statistical region is found in the (0-7)-fold (clean singles) to deviate from the exponential shape and shows a downward curvature. Comparison of these shapes with statistical model calculations are in progress to see whether these results and the deduced multipole composition in the region can be reproduced with the current concepts of the level density expressions that have been established earlier from data at considerably lower excitation energies and angular momenta.

(k) EVIDENCE FOR PRE-EQUILIBRIUM EFFECTS IN $160+154$ Sm FUSION REACTIONS K. Geoffroy, D. G. Sarantites, M. L. Halbert, R. A. Dayras, D. C. Hensley, J. H. Barker

In order to investigate the presence of pre-equilibrium particle emission in fusion reactions with heavy ions other than ${ }^{12} \mathrm{c}^{4+}$ we have measured the $\gamma$-ray multiplicities $\left\langle M_{\gamma}\right\rangle$ and cross sections for products formed in the reactions of $112,127,142,156$ and $169 \mathrm{MeV}{ }^{16} 0$ with ${ }^{154} \mathrm{Sm}$. A Ge(Li) detector in an anti-Compton arrangement was used to identify the exit channel and transition and $10 \mathrm{NaI}$ detectors $\left(2^{\prime \prime} \times 3^{\prime \prime}\right)$ were employed to record the distribution of coincidences from which the first three moments of the multiplicity distribution were extracted.

The total multiplicity and the multiplicity to specific exit channels first increases with ${ }^{16} 0$ bombardment energy and then levels off to 224 for the three highest bombardment energies. When $\langle M\rangle$. is plotted vs. $x$ for each bombardment energy saturation effects indicative of pre-equilibrium emission of neutron and $\alpha$-particles are observed at the three highest bombardment energies. 
In Fig. $3<P_{x}$ is shown vs. $x$. At the lowest two energies $\langle M\rangle_{x}$ increases essentially linearly with decreasing $x$, whereas for the highest three energies $\langle M\rangle_{x}$ first increases and then levels off with decreasing $x$. These effects are clear indications of pre-equlibrium emission (See Reports c00-4052-12 and 13).

In order to investigate these effects in more detail we have carried out a multiple coincidence experiment in which the neutron and charged particle spectra were recorded in coincidence with $\mathrm{Ge}(\mathrm{Li})$ detectors and 8 NaI detectors for the purpose of extracting spectra leading to each exit channel and at the same time deduce the associated $\gamma$-multiplicity.

For this purpose the ${ }^{16} \mathrm{O}+{ }^{154} \mathrm{Sm}$ reaction was used at $155 \mathrm{MeV}$ of bombardment energy. Neutron time-of-flight spectra were recorded with 4 neutron detectors at $20,70,120,160^{\circ}$ in coincidence with either of two $\mathrm{Ge}(\mathrm{L} I)$ detectors located at 55 and $125^{\circ}$ to the beam. Simultaneously six $\triangle \mathrm{ExE}$ light particle telescopes (with E detector.'of $1500 \mu \mathrm{m}$ ) were used to record the proton and $\alpha$-particle spectra in coincidence with the two Ge(Li) detectors. In addition the coincident events were tagged according to the number of $\mathrm{NaI}$ detectors that fired, for the purpose of extracting $\gamma$-ray multiplicity information.

The experiment was successful and 74 event-tapes of data were accumulated. These results are currently being analyzed. 
Fig. 3. Multiplicities $\langle M\rangle_{x}$ for the various $\mathrm{xn}$ products formed in the reactions of ${ }^{16} \mathrm{O}+{ }^{154} \mathrm{Sm}$ at the indicated excitation energies as a function of the number of emitted neutrons. Note the leveling of $f$ of $\langle M\rangle_{x}$ at the higher excitations. 


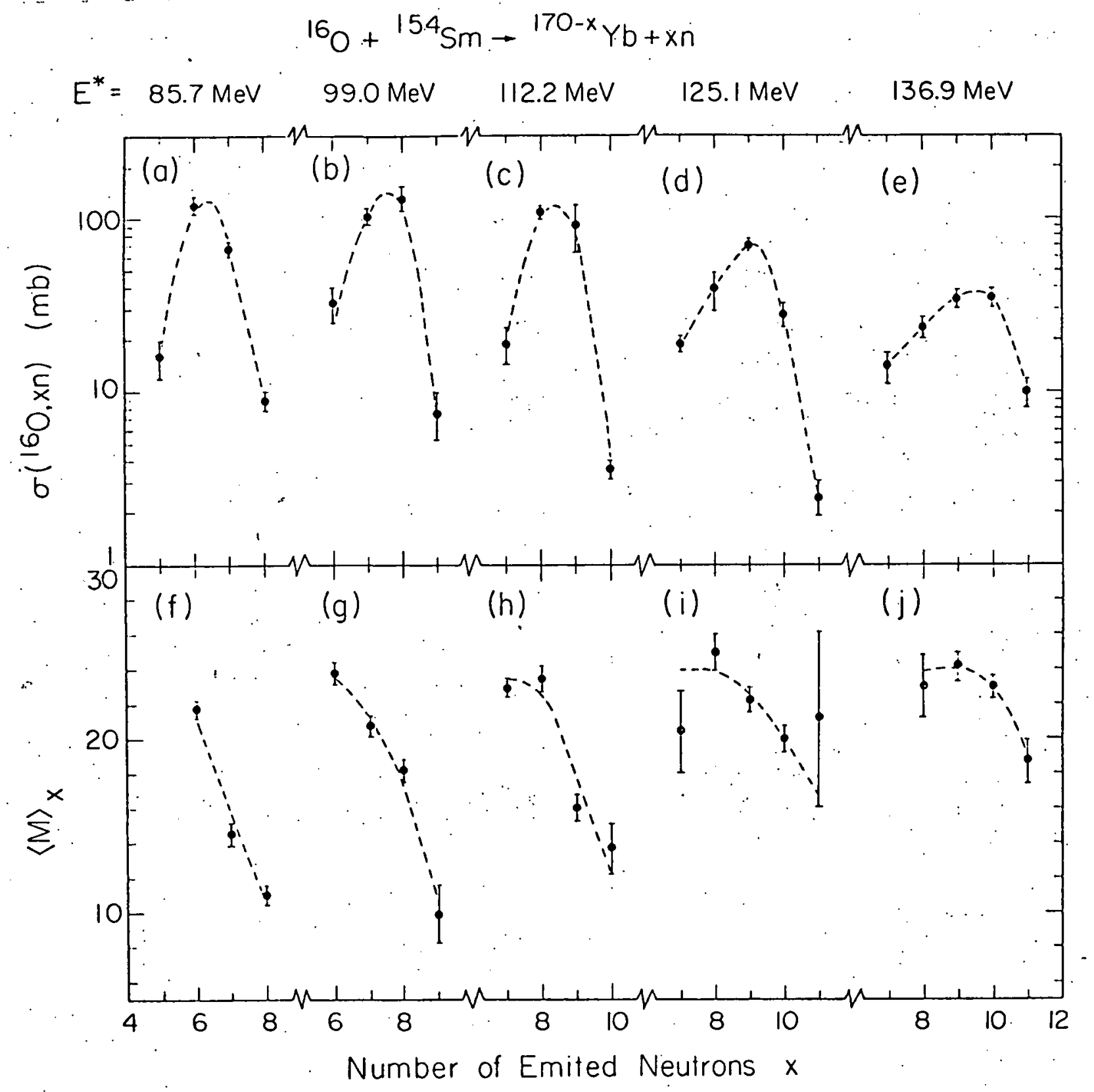

Fig. 3 
(1) GAMMA-RAY MULTIPLICITIES FROM REACTIONS FORMING THE COMPOUND NUCLEUS $100 \mathrm{Ru}$

In order to Investigate the deviations from the compound nucleus behavior at lower excitations as evidenced by means of the saturation of the $\gamma$-ray multiplicity we have employed the ${ }^{4} \mathrm{He}+{ }^{96} \mathrm{Mo} \rightarrow{ }^{100-x_{\mathrm{Ru}}}+\mathrm{xn}$ reactions for Initial excitations of $21.1,26.9$ and $31.7 \mathrm{MeV}$ in $100 \mathrm{Ru}$. The experiments were carried out at the Washington University cyclotron. In the upper part of Fig. 4 are shown the cross-sections $\sigma_{x}$ plotted versus the number of emitted neutrons $x$. In the lower part the total $\gamma$-ray multiplicites are shown as the data points. The solid lines are theoretical curves calculated using the evaporation code GROGI. The agreement is satisfactory except for the highest bombardment energy vihere a saturation in $\langle M\rangle_{x}$ is observed for $x=1$ which is not predicted by the statistical theory. 
Fig. 4. Total cross sections $\sigma_{x}$ and multiplicities $\langle M\rangle_{x}$ for the various xn products formed in the reactions of ${ }^{4} \mathrm{He}+{ }^{96} \mathrm{Mo}$. The lines are the theoretical curves calculated on the basis of the statistical model. The expected essentialiy linear dependence is : not obeyed by the data of the highest energy which indicate preequilibrium effects for the $x=1$ channel. 


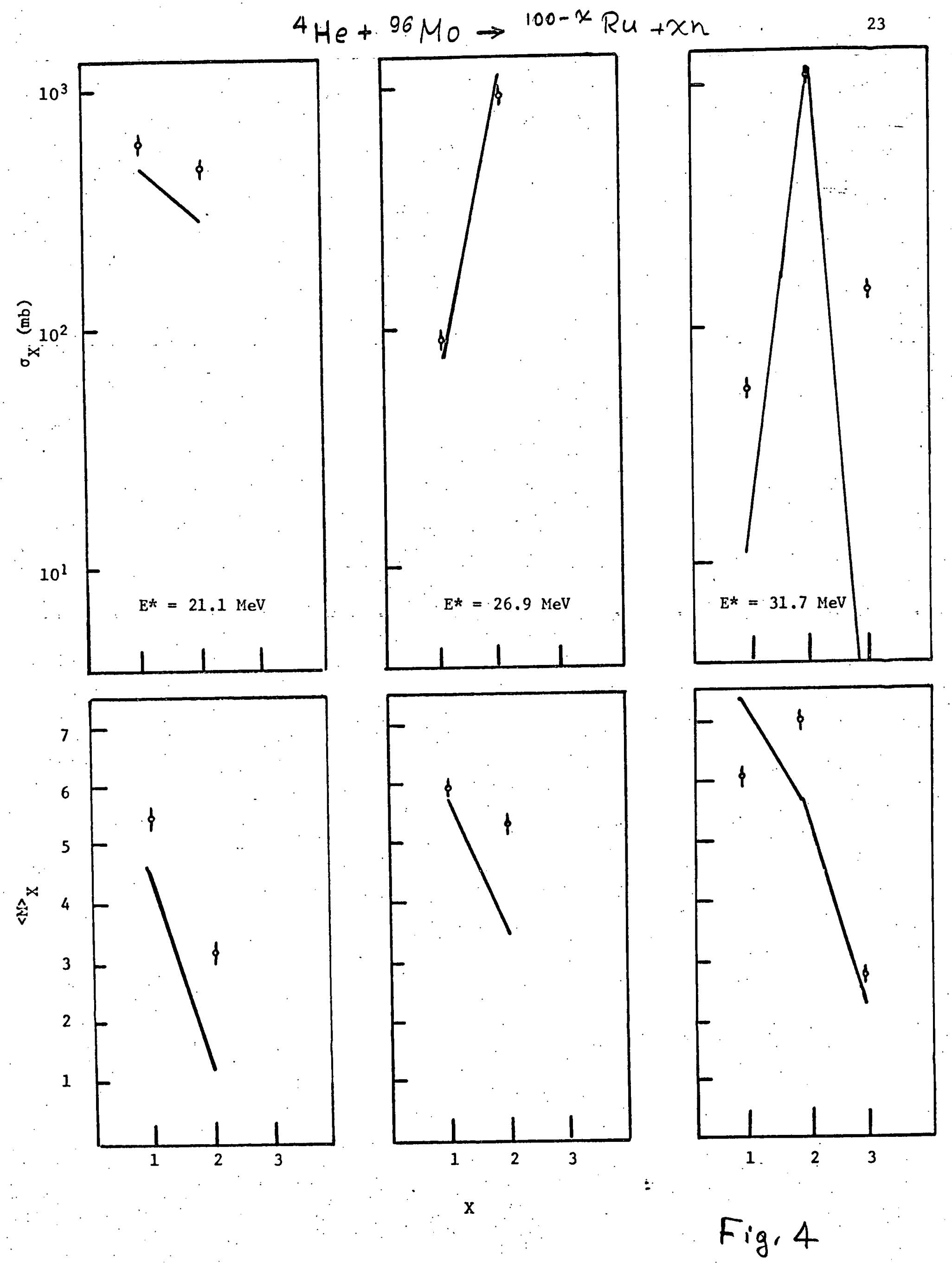


(m) MULTIPLICITIES OF $\gamma$-TRANSITIONS OBSERVED IN ${ }^{6} \mathrm{Li}$ INDUCED REACTIONS

D. G. Sarantites, L. Westerberg, H. Karwowski, ${ }^{*}$ J. D. Wiggins, ${ }^{*}$ P. P. Singh, * M. E. Sadler, ${ }^{*}$ and J. Jastrzebski *

The apparatus for $\gamma$-ray multiplicity experiments, constructed at Washington University, St. Louis, was transported to IUCF for the experiments and was set-up in the $\gamma$-cave. It consisted of two lead shields which support seven and two $2^{\prime \prime} \times 3^{\prime \prime} \mathrm{NaI}$ detectors, respectively. The smaller of the two also allows positioning of a $\dot{\mathrm{Ge}}(\mathrm{Li})$ detector at $90^{\circ}, 5 \mathrm{~cm}$ from the target.

In the first experiments $66 \mathrm{MeV}$ of ${ }^{6} \mathrm{Li}$ beams bombarded targets of ${ }^{56} \mathrm{Fe}$, ${ }^{165} \mathrm{Ho}$ and ${ }^{169} \mathrm{Tm}$. In these experiments $\mathrm{Ge}(\mathrm{Li})$ spectra were recorded on magnetic tape in the event-by-event mode in coincidence with $0,1,2,3$ or more NaI detectors. Energy spectra in the NaI detectors at $50^{\circ}, 90^{\circ}, 135^{\circ}$ and $140^{\circ}$ were also recorded in coincidence with the Ge(Li) pulses.

For the ${ }^{56} \mathrm{Fe}$ target the average multiplicity, $\langle\mathrm{M}\rangle$, is about 8.5 for transitions in $\mathrm{Ni}$ isotopes but it is only $\sim 3.5$ for transitions in nuclei. close to the target, namely ${ }^{56} \mathrm{Fe},{ }^{57} \mathrm{Fe}$ and ${ }^{57} \mathrm{Co}$. For the remainder products $<M>$ varies between 4 and 5 .

For the ${ }^{165}$ Ho target the $\langle M\rangle$ values vary with $x$ from $13,15,13$ and 11 for $x$ of $4,5,6$, and 7 , respectively. These results indicate that at this bombardment energy deviations from equilibrated compound nucleus formation has been observed.

Further experiments at higher energies have been planned.

*Indiana University, Department of Physics. 
(n) $\gamma$-RAY MULTIPLICITES IN REACTION PRODUCTS FROM. $80 \mathrm{MeV}$ PROTON BOMBARDMENTS OF ${ }^{62} \mathrm{Ni}$ AND ${ }^{169} \mathrm{Tm}$ J. D. Wiggins, * M. E. Sadler, ${ }^{*}$ H Karwowski, ${ }^{*}$ J. Jastrzebski, ${ }^{*}$ P. P. Singh, * L. Westerberg and D. G. Sarantites

Preliminary analysis of the average $\gamma$-ray multiplicites indicate that for reaction of $80 \mathrm{MeV}$ proton with ${ }^{52} \mathrm{Ni}$ they range from a value of about 3 for products close to the target to a value of about 6 for nuclei 8-10 mass units away from the target mass.

*Indiana University, Physics Department. 


\subsection{ST-RONGLY DAMPED HEAVY-ION COLLISIONS}

(a) Angular Momentum Transfer in Deep Inelastic Scattering

R. A. Dayras, ${ }^{*}$ R. G. Stokstad, ${ }^{*}$ C.B. Fulmer, ${ }^{*}$ M. L. Halbert,, D. C. Hensley, ${ }^{*}$ R. L. Robinson, * A. H. Snell, ${ }^{* *}$ L. Westerberg and D. G.

\section{Sarantites}

Detailed measurements have been made of the first three moments (average, width and skewness) of the $\gamma$-ray multiplicity distributions, in coincidence with the projectile-like fragments and the evaporation residues produced in the bombardment of ${ }^{63} \mathrm{Cu}$ by $165 \mathrm{MeV}{ }^{20} \mathrm{Ne}$ and $130 \mathrm{MeV}{ }^{12} \mathrm{C}$ ions. From those measurements, the angular momentum imparted to the fragments during the collision has been determined and comparison is made with current models. The implications of the broad width of the angular momentum distribution following deeply inelastic collisions are discussed.

The study of strongly damped collisions for a variety of nuclei has led to the concept of a short-lived, rotating dinuclear complex which, after a large damping of the initial kinetic energy of relative motion separates into projectile-like and target-like fragments. It is customarily assumed that the fragments share, according to their relative masses, an excitation energy determined by the degree of damping of the initial relative motion.

Until now, experimental information was mainly obtained from the characteristics of the final reaction products which are easily measured: kinetic energy, mass, charge, angular distribution and cross-section. From these measurements, it has been established that energy damping and mass exchange increase with the rotation (or lifetime) of the dinuclear complex

*Oak Ridge National Laboratory, Physics Division. ** Consultant with the Oak Ridge National Laboratory, Physics Division. 
before decay. These aspects of strongly damped collisions are relatively well understood under a classical description of the equations of motion Including dissipative terms and a diffusion process based on the FokkerP1ank equation.

However the preceeding measurements address themselves only indirectly to the nature of the nuclear complex formed during the collision by establishing the strength of the radial friction which converts the energy of relative motion into excitation energy of the intrinsic degrees of freedom, The measurements of the angular momentum imparted to the final fragments probe directly the role played by tangential friction during the collision. Such measurements can determine if these frictional forces are sufficient to lead to the formation of a rigid body within the collision time or whether the colliding nuclei preserve some relative motion at the scission time.

\section{Experimental Set-up}

Beams of $130 \mathrm{MeV}{ }^{12} \mathrm{C}$ and $165 \mathrm{MeV}{ }^{20} \mathrm{Ne}$ ions, from the 0ak Ridge Iso-. chronous Cyclotron bombarded $1 \mathrm{mg} / \mathrm{cm}^{2}$ targets of $99 \%{ }^{63} \mathrm{Cu}$. A set of $9 \mathrm{NaI}(\mathrm{T} 1$ ) detectors (2 " $\times 3 "$ ") was operated in coincidence with two heavy ion telescopes consisting of a $\Delta \mathrm{E}$ gas ionization chamber and a $1500 \mu \mathrm{m}$ surface barrier Edetector (see Fig. 5). The front faces of the individually shielded NaI detectors were located $10 \mathrm{~cm}$ from the target and their thresholds were set at $85 \mathrm{keV}$. The HI counter telescopes were located on each side of the beam. One was used to cover the angular range between $10^{\circ}$ and $30^{\circ}$ and subtended a solid angle of $3 \mathrm{mstr}$. The other one was used to cover the angular range between 30 and $60^{\circ}$ and subtended a solid angle of 9 mstr. The counting rates in both detectors were kept approximately equal by an appropriate 
Fig: 5 Experimental arrangement. 
SET - UP

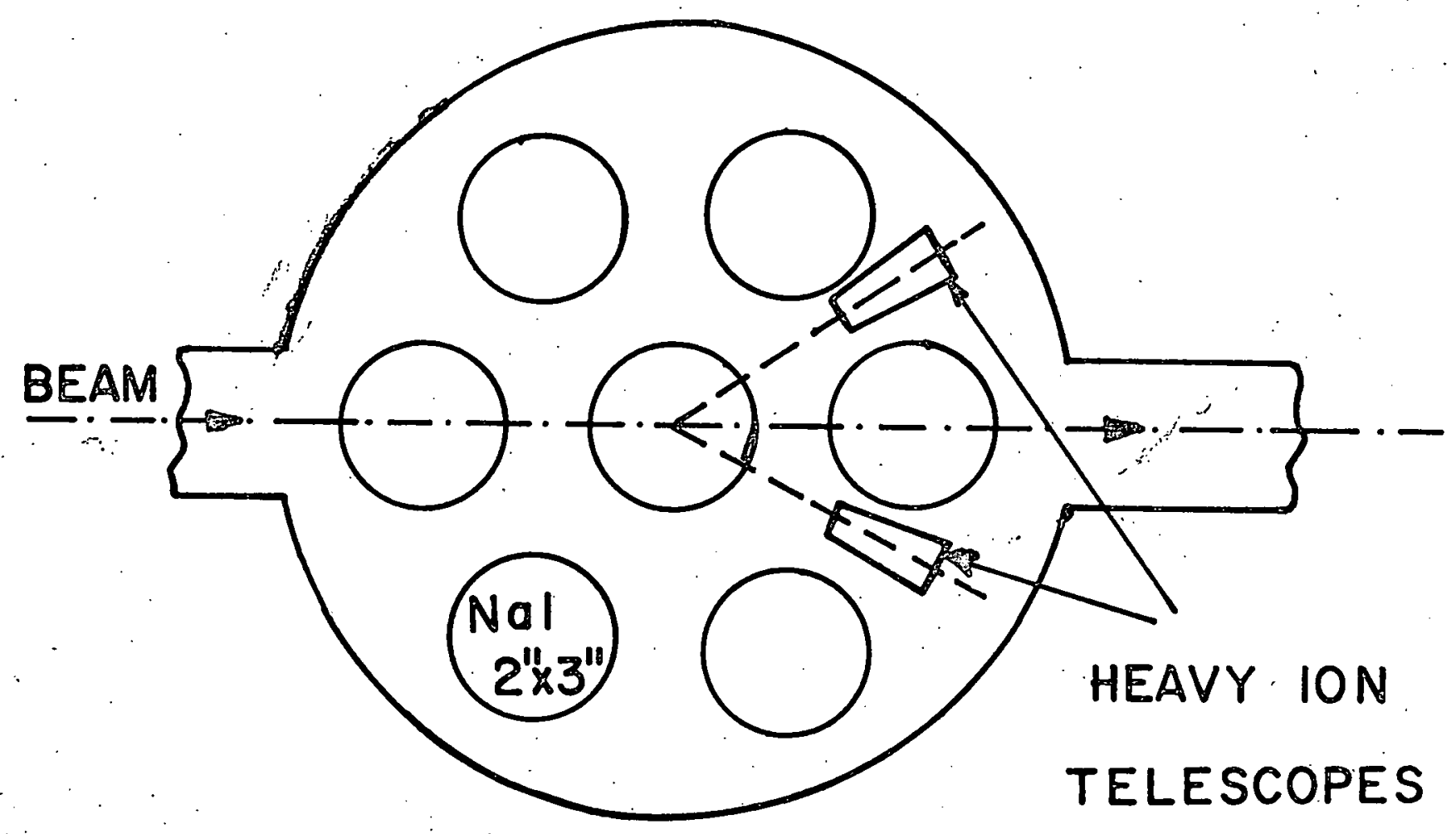

Fig. 5 
choice of their relative position.

Great care has been exercised to minimize the possible presence of light contaminants on the targets (carbon build up, oxidation of the target). To decrease the presence of hydrocarbons in the thoroughly cleaned reaction chamber, the vacuum was maintained to $\simeq 2 \cdot 10^{-6}$ torr with a high capacity cryopump $(1400 \mathrm{l} / \mathrm{s})$. All vacuum seals were made using low-vapor pressure vacuum grease. Shortly before use each copper target was reduced under hydrogen atmosphere. Furthermore, during a run the target was periodically moved as to bombard the same spot for only a short time ( $\leq 1 \mathrm{hr}$ per position). In order to check for possible presence of light contaminants, each run was repeated with a carbon target and the spectral shapes of the low $\mathrm{Z}$ products were compared.

For a particular angular setting, depending on whether $0,1,2,3$ or 4 or more NaI detectors recorded $\gamma$ rays in coincidence with one of the heavyIon telescopes, the digitized $\Delta E$ and $E$ signals were stored on-line in one of five $200 \times 300$ channel arrays. A similar set of five arrays was stored for the other telescope. Simultaneously, and in coincidence with the heavy-ion telescopes, the linear signal from 4 of the $9 \mathrm{NaI}$ detectors were recorded event-by-event on magnetic tape. Two of these NaI detectors were in the reaction $\mathrm{plane}$ at $\theta=145^{\circ}$ and $\theta=125^{\circ}$ relative to the beam direct1on. The other two detectors were located out of the reaction plane at $\theta=$ $90^{\circ}, \phi=0$ and $\theta=111^{\circ}, \phi=45^{\circ}$ where $\phi$ is the angle between the perpendicular to the reaction plane and the detectors.

In order to estimate the effect. of neutron detection on the extracted $\gamma$-ray multiplicites, a separate measurement was made of the relative yield for neutrons and $\gamma$ rays by a time-of-flight measurement in one of the NaI detectors removed to 1 meter from the target at $45^{\circ}$ in the reaction plane. 
This indicated that neutron detection in the NaI detectors contributes less than 0.5 units to the deduced $\gamma$-ray multiplicites $\overline{\mathrm{M}}_{\gamma}$.

A pulser triggered externally by the beam integrator was injected into each telescope. Its yield in the $\triangle E x E$ map with different order was used to correct for random events. This correction was generally found to be smaller than $10 \%$.

To absorb the $x$-rays from the target and the lead shield surrounding the detectors, $0.5 \mathrm{~mm} \mathrm{Cd}$ and $0.13 \mathrm{~mm} \mathrm{Cu}$ absorbers were placed in front of each NaI detector. These absorbers also made the total detection efficiency $\Omega$ less dependent upon the $\gamma$-ray energies. The NaI detectors were calibrated in-situ with standard sources of ${ }^{107} \mathrm{Cd},{ }^{57} \mathrm{Co},{ }^{133} \mathrm{Ba},{ }^{137} \mathrm{Cs}$ and ${ }^{60} \mathrm{Co}$. The average detection efficiency for NaI detectors was found to be 0.00465 , $0.00945,0.01119,0.00933$, and 0.00844 at $88,124,276,662$ and $1253 \mathrm{keV}$ respectively. Above $500 \mathrm{keV}$, due to the increasing effect of multiscatterIng from the lead shield, the detection efficiency remained practically constant.

Coincidences between the heavy-ion telescopes and the individual NaI detectors was established by the overlap coincidence technique. To take into account the different arrival times in the telescopes of ions with different mass and energy a resolving time $2 \tau \simeq 100$ ns was found optimum.

Measurements were done with the telescopes located at $15^{\circ}, 20^{\circ}, 25^{\circ}$, $30^{\circ}, 40^{\circ}$ and $50^{\circ}$ in ${ }^{12} \mathrm{C}$ bombardments and at $20^{\circ}, 26^{\circ}, 35^{\circ}$ and $45^{\circ}$ in the $20 \mathrm{Ne}$ bombardment.

Special measurements were made to determine: the $\gamma$-ray multiplicity of the evaporation residues. In order to detect the evaporation residues which emerge with low energy, the gas pressure in the $\Delta \mathrm{E}$ ionization chamber was decreased by a factor of 2 and the $E$ and $\Delta E$ again adjusted so as to 
see the complete evaporation residue spectrum. Measurements were done at $16^{\circ}, 20^{\circ}$ and $30^{\circ}$ in the ${ }^{12} \mathrm{C}$ bombardment and $10^{\circ}, 15^{\circ}, 20^{\circ}$ and $28^{\circ}$ in the ${ }^{20} \mathrm{Ne}$ bombardment. These measurements are summarized in Table 1.

Table 1

Experimental Conditions

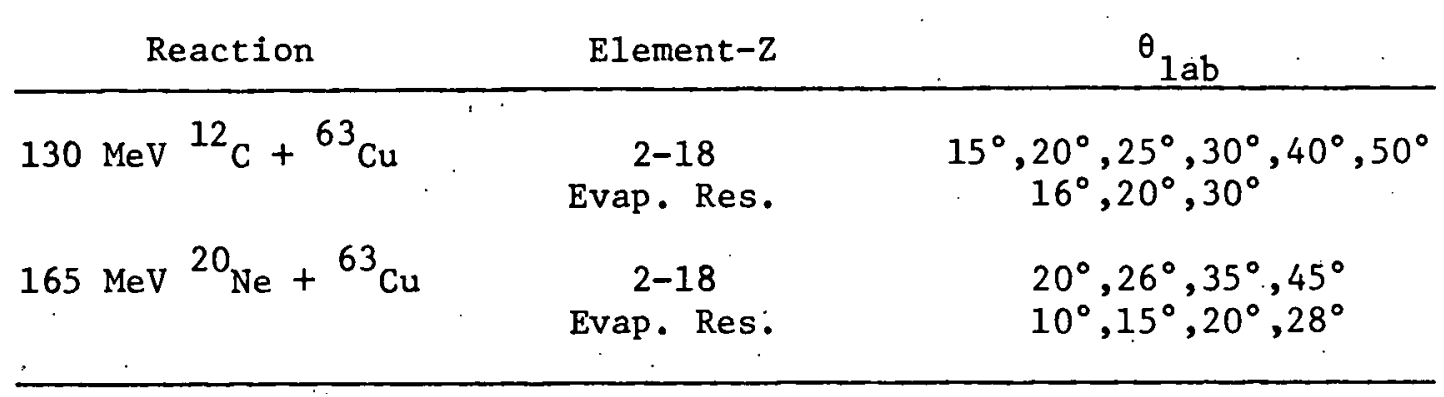

\section{Experimental Results}

An energy spectrum was obtained for each individual $\mathrm{z}$ by drawing a curved gate around each element of the $\triangle \mathrm{ExE}$ maps and then projecting its content on the energy axis. For a given element $\mathrm{Z}$, the same gate was used to obtain. the energy spectra corresponding to coincidences at different order. Figure 6 shows the different fold spectra for carbon fragments emitted at $35^{\circ}$ in the reaction ${ }^{20} \mathrm{Ne}+{ }^{63} \mathrm{Cu}$ at $165 \mathrm{MeV}$. For this element far remote from the projectile the spectrum is dominated by the deep inelastic component. The presence of a given elastic contribution is indicated only by a high energy tail.

To extract the $\gamma$-ray multiplicities as a function of the energy of the projectile-like fragments, the energy spectra were divided in energy bins. Then, the first three moments (average, width and skewness) of the multiplicity distribution were determined for a given energy of the projectile-like fragment from the distribution of the counts in the 
Fig. 6. Carbon spectra from the reaction ${ }^{20} \mathrm{Ne}+{ }^{63} \mathrm{Cu}$ recorded with a heavy-ion telescope in multiple coincidence with $9 \mathrm{NaI}(\mathrm{T} 1)$ detectors. The 0 -fold refers to the absence of coincidence and the higher fold refers to the sum of 4 to 9 -fold coincidences. The single spectrum is the sum of the different folds. 
${ }^{20} \mathrm{Ne}+{ }^{63} \mathrm{Cu} \rightarrow$ Carbon

$$
\theta=35^{\circ}
$$

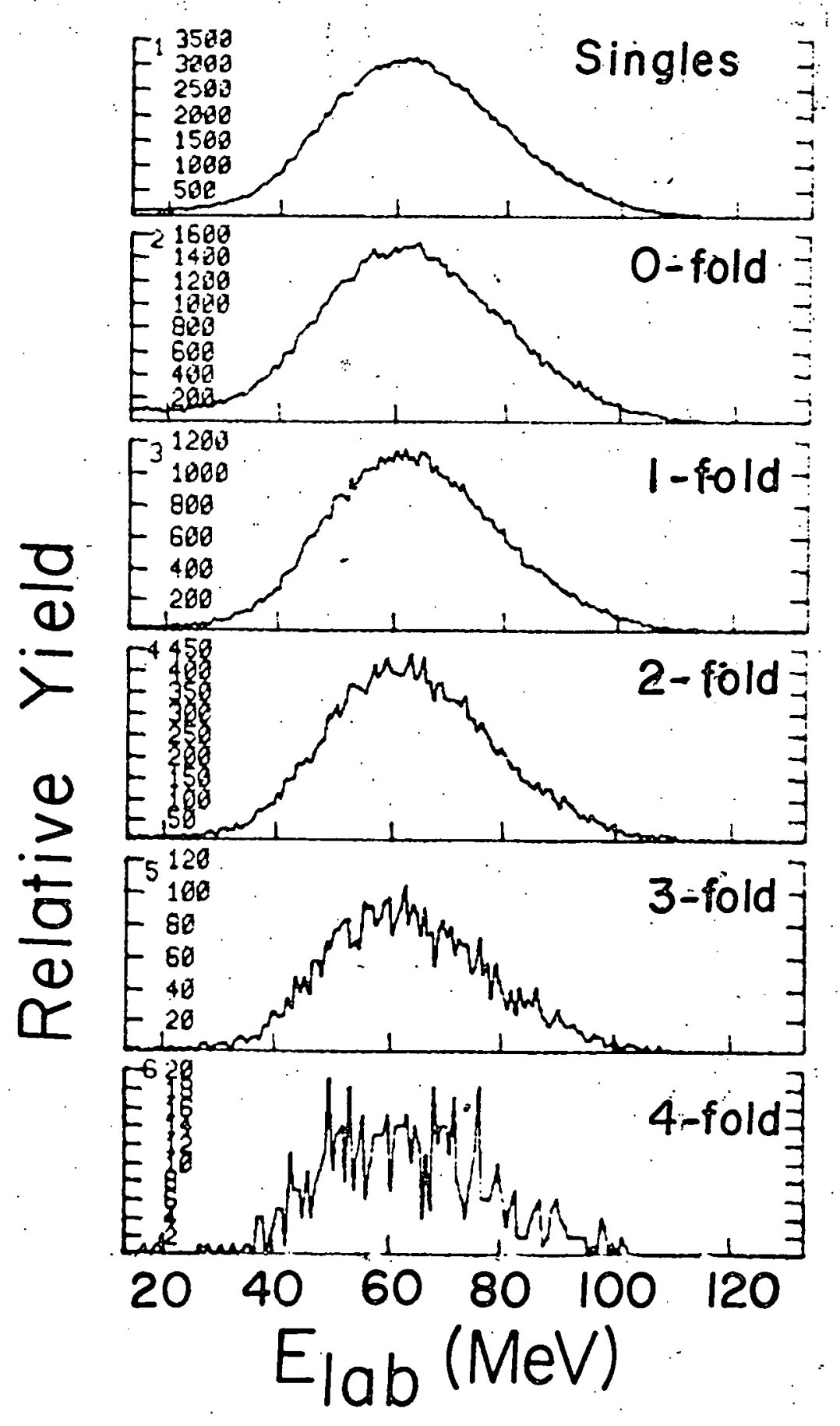

Fig. 6 
corresponding energy line in the different fold spectra. The width, $\sigma$, of the multiplicity distribution is defined by the relation $\sigma^{2}=\left\langle(M-\bar{M})^{2}\right\rangle$ and the skewness $s$ by the relation $s=\frac{\left\langle(M-\bar{M})^{3}\right\rangle}{\sigma^{3}}$ where $\bar{M}$ denotes the average multiplicity. These quantites can be determined from the counts observed in the different folds. A detailed treatment of this problem has been presented elsewhere. In the present case,..the...average.multiplicity $\bar{M}_{\gamma}$ can be written as:

$$
\begin{aligned}
\bar{M}_{\gamma}= & \frac{1}{N \bar{\Omega}}\left\{\mathrm{P}_{N_{1}}+\frac{2 N-1}{N-1} \mathrm{P}_{2}+\frac{3 N^{2}-6 N+2}{(N-1)(N-2)} \mathrm{P}_{\mathrm{N}_{3}}\right. \\
& \left.+\frac{2\left(2 \mathrm{~N}^{3}-9 \mathrm{~N}^{2}+11 N-3\right.}{(\mathrm{N}-1)(\mathrm{N}-2)(\mathrm{N}-3)} \mathrm{P}_{N_{4}}+\ldots\right\}
\end{aligned}
$$

where $\mathrm{N}=$ number of detectors

$\bar{\Omega}=\gamma$-ray average total efficiency

and $P_{N I}=\frac{C i}{\sum_{j=0}^{N} C_{j}}$ where $C_{i}=$ number of counts in the $i-f o l d$ spectrum

In the present case, as can be seen from Fig. 6, the terms of order 5 and higher can be neglected. The previous expression neglects the effect of neutron detection and the angular correlations between the $\gamma$-rays. As has already been "mentioned the neutron detection contributes at most 0.5 units to the average multiplicity and has been neglected. From the measurements in and out of the reaction plane, the anisotropy in the $\gamma$-ray angular. distribution does not exceed $20 \%$. Furthermore, due to the position of the different NaI detectors, this anisotropy in the $\gamma$-ray does not affect the measured multiplicities by more than $5 \%$.

As can be seen from relation (1), in order to extract the average 
multiplcity $\bar{M}_{\gamma}$ and the other moments as well, it is necessary to know the average $\gamma$-ray energy. This information has been obtained by unfolding the NaI spectra obtained in coincidence with the projectile-like fragments. For both reactions, ${ }^{20} \mathrm{C}+{ }^{63} \mathrm{Cu}$ and ${ }^{20} \mathrm{Ne}+{ }^{63} \mathrm{Cu}$, the average $\gamma$-ray energies vary between 1.8 and $2.2 \mathrm{MeV}$ and seem to be independent of the $\mathrm{Z}$ of the fragment and of its energy. 'In'this energy'range, the NaI average effi-' ciency varies by less than $2 \%$.

Fig. 7 shows the dependence of $\bar{M}_{\gamma}$ upon the energy of the projectilelike fragment in the reaction ${ }^{20} \mathrm{Ne}+{ }^{63} \mathrm{Cu}$. The sodium spectrum show clearly two components. The high energy component is associated with quasi-elastic events. The low energy component corresponds to strongly damped collisions. For the fragments with a $\mathrm{Z}$ more remote from the $\mathrm{Z}$ of the projectile, the spectra are dominated by strongly damped collisions. In each case, the r-ray multiplicity $\bar{M}_{\gamma}$ shows the same behavior. $\bar{M}_{\gamma}$ increases with the energy lost in the collision to reach a maximum in the region of the peak of the deep inelastic scattering. Then as the energy loss continues to increase $\bar{M}_{\gamma}$ decreases sharply. A similar behavior is observed in the reaction ${ }^{12} \mathrm{C}+{ }^{63} \mathrm{Cu}$ (Fig. 8).

The measured values of $\bar{M}_{\gamma}$ have to be related to the initial fragment spins. The $\gamma$-ray cascades can be divided in two parts: i) the statistical transitions which proceed primarly from the entry states populated by partial emission, toward the yrast line. These $\gamma$-rays are dipolar in value and in average carry almost no angular momentum. ii) The remaining transitions follow a path parallel to yrast line and are responsible for angular momentum dissipation. These transitions are stretched quadrupole in nature. It is often assumed that the angular momentum carried out by particle emission and the presence of the statistical $\gamma$-ray compensate one another and the 
Fig. 7. Dependence of $\bar{M}_{\gamma}$ on the energy of the projectile-1ike fragment in the reaction ${ }^{20} \mathrm{Ne}+{ }^{63} \mathrm{Cu}$. The black dots are the energy spectra. The low energy shaded area corresponds to low energy non-identified heavy-ions (mainly evaporation residues) which were included in the curved gates drawn on $\Delta$ ExE maps. Two components can be clearly seen in the sodium spectrum $(z=11)$. The high energy component corresponds to quasi-elastic collisions. The low-energy component comes from strongly damped collisions. For nitrogen and magnesium, the spectra are dominated by strongly damped collisions. The squares represent the $\gamma$ ray multiplicity for a given erergy of the fragments. The lines through the squares are only to guide the eye. 

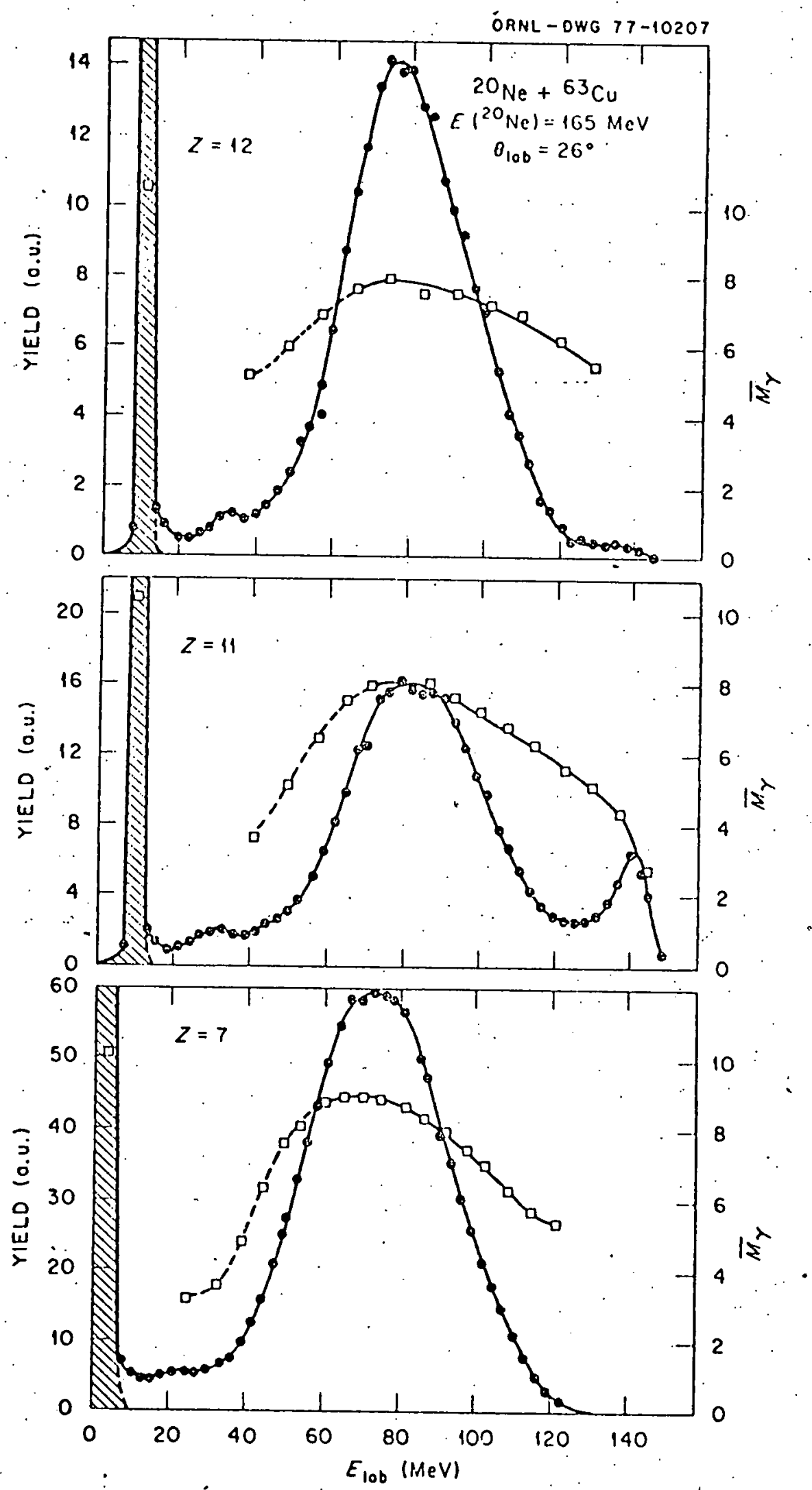

Fig. 7 
Fig. 8. Depedence of $\overline{\mathrm{M}}_{\gamma}$ on the projectile-like fragment in the reaction ${ }^{12} \mathrm{C}+{ }^{63} \mathrm{Cu}$. 
$130 \mathrm{MeV}{ }^{12} \mathrm{C}+{ }^{63} \mathrm{Cu} \quad \theta_{\text {lab }}=50^{\circ}$

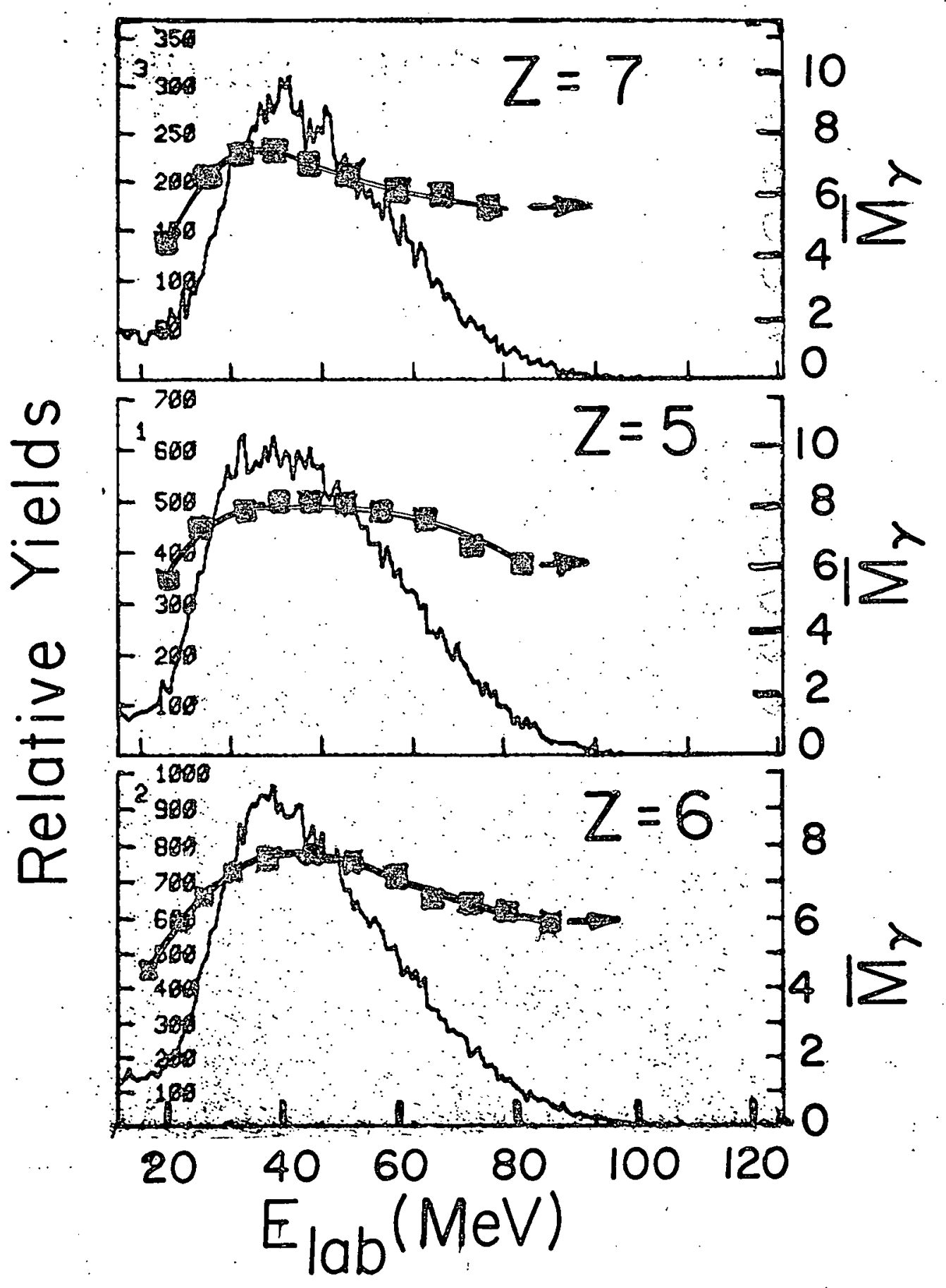

Fig. 8 
initial angular momentum I is related to the measured multiplicity $\bar{M}_{\gamma}$ by the relation $I=2 \bar{M}_{\gamma}$. This relation is approximately correct for heavy compound systems which decay mainly by the emission of neutrons which carry a very small amount of angular momentum. However for lighter systems like the one under consideration, the emission of charged particles can play an important role in angular momentum dissipation.

In order to establish a relation between angular momentum and multiplicity, we have measured the $\gamma$-ray multiplicities for the evaporation residues from the reactions ${ }^{12} \mathrm{C}+{ }^{63} \mathrm{Cu}$ and ${ }^{20} \mathrm{Ne}+{ }^{63} \mathrm{Cu}$. The evaporation residue cross-sections which in the absence of fission are identical to the fusion. crosssections, have been measured independently for those reactions (Table 2). From these measurements, we have extracted a critical angular momentum $\ell_{c r}$ for fusion of $51 \%$ and $56 \hbar$ for the ${ }^{12} \mathrm{C}+{ }^{63} \mathrm{Cu}$ and ${ }^{20} \mathrm{Ne}+{ }^{63} \mathrm{Cu}$ respectively. From these values, assuming a triangular distribution of angular momenta, we obtain an average angular moment $\bar{\ell}=\frac{2}{3} \ell_{c r}$ in the compound nuclei of $34 \mathrm{~h}$ and $37.3 \mathrm{~h}$ respectively.

\section{Table 2}

Parameters Characterizing the Reaction ${ }_{7}^{12} \mathrm{C}+{ }^{63} \mathrm{Cu}$ and ${ }^{20} \mathrm{Ne}+{ }^{63} \mathrm{Cu}$ and $130 \mathrm{MeV}$ and $165 \mathrm{MeV}$ Respectively.

$$
{ }^{12} \mathrm{C}+{ }^{63} \mathrm{Cu} \quad{ }^{20} \mathrm{Ne}+{ }^{63} \mathrm{Cu}
$$

$\theta_{\frac{1}{4}} 1 \mathrm{ab}$

$\theta_{\frac{1}{4} \text { C.M. }}$.

$\ell_{\text {max }}$

${ }^{\ell}$ cr

$\sigma_{\mathrm{R}}$

$\sigma_{f}$

$\sigma_{\mathrm{DI}+\mathrm{QE}}$
13.6

16.1

58.6 *

51 光

$2107 \mathrm{mb}$

$1800 \mathrm{mb}$

$300 \mathrm{mb}$
18.1

24.3

71.6 h

$56 \pi$

$1930 \mathrm{mb}$

$1128 \mathrm{mb}$

$802 \mathrm{mb}$ 
Fig. ' 9 summarizes our multiplicity measurements for the evaporation residues from the reactions ${ }^{12} \mathrm{C}+{ }^{63} \mathrm{Cu}$ and ${ }^{20} \mathrm{Ne}+{ }^{63} \mathrm{Cu}$. In both cases, the multiplicity $\bar{M}_{\gamma}$ drops with the detection angle. This effect is the manifestation of the effect of particle evaporation on the $\gamma$-ray multiplicities. These evaporation residues which are detected at larger angles have received a larger transverse momentum than those detected at more forward angles. This is the result of the emission of more energetic particles which carry also a larger angular momentum, causing a drop in the measured multiplicities. Extrapolating to $0^{\circ}$ detection angle, we find $\overline{\mathrm{M}}_{\gamma}=9.9$ and 11.6 for ${ }^{12} \mathrm{C}+{ }^{63} \mathrm{Cu}$ and ${ }^{20} \mathrm{Ne}+{ }^{63} \mathrm{Cu}$ respectively. Using the relation $\bar{l}=2 \overline{\mathrm{M}}_{\gamma}$, we obtain $\bar{l}=19.8$ and 23.2 for the average angular momenta brought into the compound nuclei $75 \mathrm{Br}$ and $83_{\mathrm{Y}}$ respectively. These values are 214 smaller than values obtained from cross-section measurements. This extra angular momentum is carried out by particle emission. Using these measurements and measurements of $\overline{\mathrm{M}}_{\gamma}$ for $\mathrm{Cu}$ compound nuclei formed in the reactions $\alpha+\mathrm{Co}$ and ${ }^{12} \mathrm{C}+\mathrm{V}$, we have established an empirical relation between $\overline{\mathrm{M}}_{\gamma}$ and $\bar{l}$.

$$
\bar{\ell}(E)=\bar{\ell}\left(E_{0}\right)+2\left[M_{Y}^{-}(E)-\bar{M}_{\gamma}\left(E_{0}\right)\right]+\frac{3}{16}\left(E-E_{o}\right)
$$

where $E_{o}$ is the excitation energy at which the multiplicity $\bar{M}_{\gamma}\left(E_{0}\right)$ and the average angular momentum $\bar{\ell}\left(E_{0}\right)$ have been measured independently. $E$ is the actual exctiation energy at which we have measured the multiplicity $\bar{M}_{\gamma}(E)$; $\bar{l}(E)$ is the average angular momentum of the system before particle emission. The last term in expression (2) takes into account the angular momentum carried out by particle emission which increases approximately linearly with the excitation energy.

Figure 10 shows the angular momentum transferred to the fragments in deeply inelastic collisions form the reaction ${ }^{20} \mathrm{Ne}+{ }^{63} \mathrm{Cu}$, deduced from 
Fig. 9. $\gamma$-Ray multiplcity $\bar{M}_{\gamma}$ as a function of the detection angle for evaporation residues from ${ }^{20} \mathrm{Ne}+{ }^{63} \mathrm{Cu}$ and ${ }^{12} \mathrm{C}+{ }^{63} \mathrm{Cu}$. 
Evaporation Residues

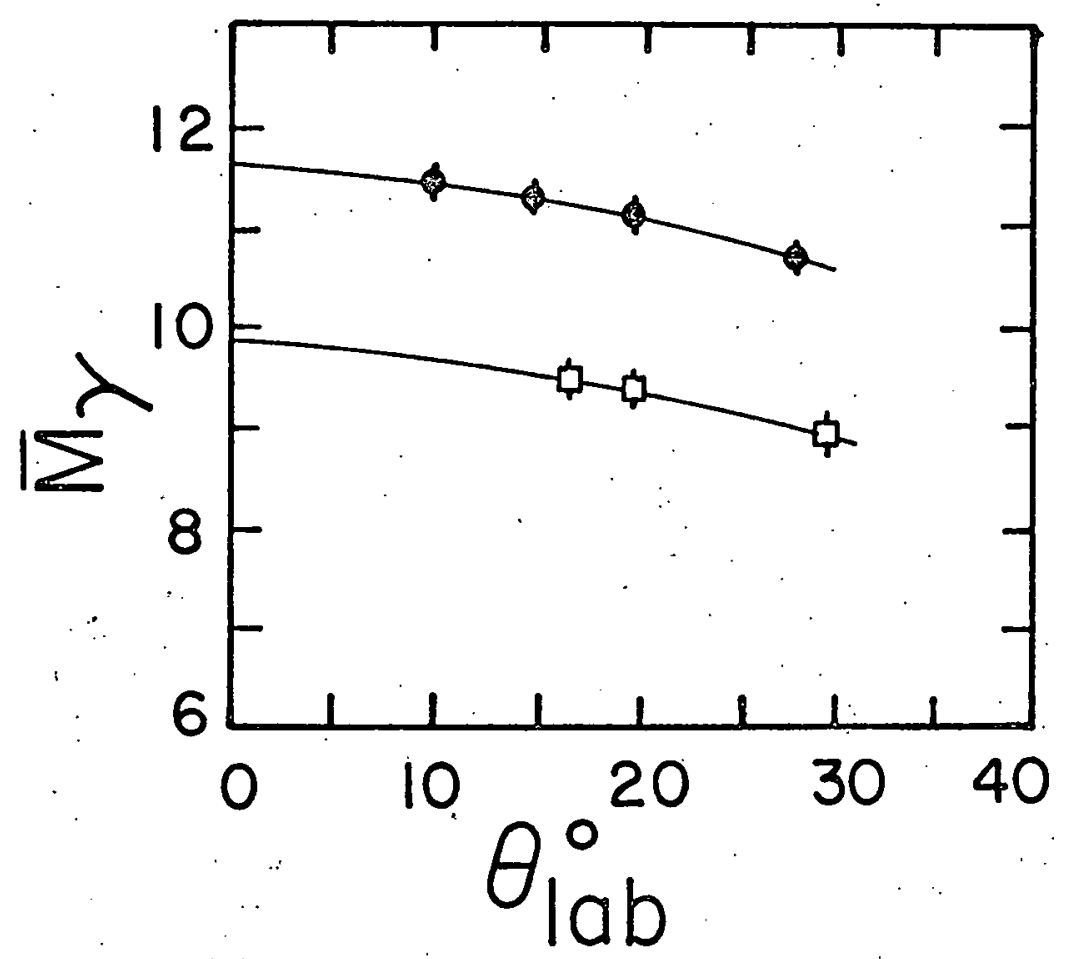

- ${ }^{20} \mathrm{Ne}+{ }^{63} \mathrm{Cu}$

․ ${ }^{12} \mathrm{C}+{ }^{63} \mathrm{Cu}$

Fig. 9 
Fig. 10. Angular momentum transferred to the fragment in deeply inelastic collisions from the reaction of ${ }^{20} \mathrm{Ne}+{ }^{63} \mathrm{Cu}$ (see text).

assuming $I=2 \bar{M}_{\gamma}$

,

$\triangle$ using relation (2) 


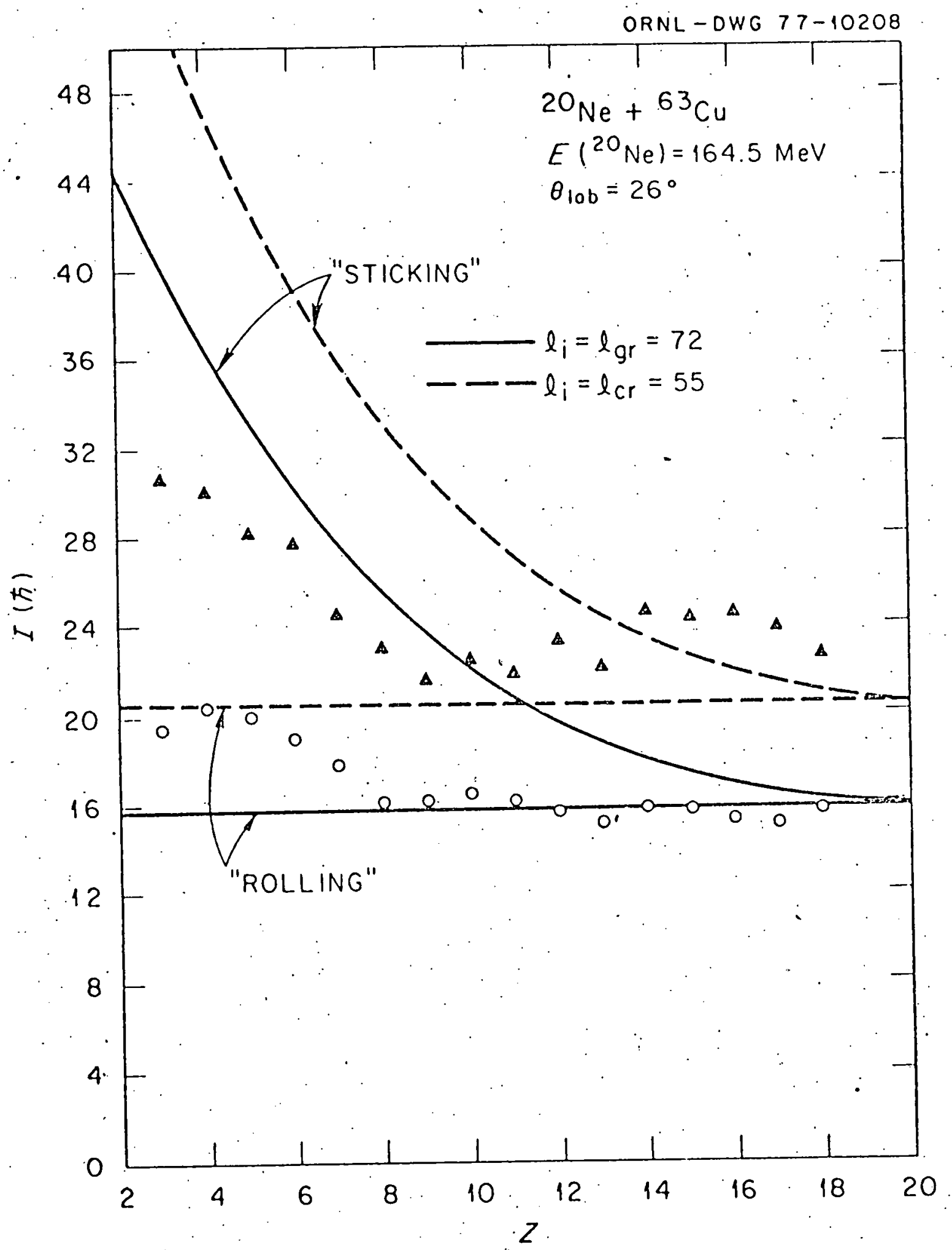

Fig. 10 
from the multiplicity measurements, as a function of the $Z$ of the projectile-

like fragments. The open circles have been obtained by assuming $I=2 \bar{M}_{\gamma}$, the fuli triangles have been obtained using relation (2). For a given $Z$, the difference between triangles and circles is the angular momentum taken away by particle emission. Particle emission plays an even more important role in the energy dissipation as can be seen from Fig. 11. For strongly damped collisions, only $\sim 25 \%$ of the energy is dissipated by $\gamma$-ray emission, the remaining $75 \%$ are dissipated by particle emission.

\section{Comparison with a Classical Model}

For the systems under consideration, deeply inelastic and quasi-elastic collisions are limited to a narrow band of partial waves between the critical angular momentum $\ell_{\mathrm{cr}}$ for fusion and the grazing angular momentum ID between $51 \hbar$ and $59 \hbar$ for ${ }^{12} \mathrm{C}+{ }^{63} \mathrm{Cu}$ and between $56 \hbar$ and $72 \hbar$ for ${ }^{20} \mathrm{Ne}+{ }^{63} \mathrm{Cu}$.

The collisions leading to quasi-elastic and deep inelastic scattering can be decomposed in three stages. 1) In the initial stage the two nuclei slide on each other. ii) The viscous forces create a sliding friction which exerts a torque on the nuclei and puts them into rotation until the peripheral velocities are matched and the system reaches the rolling stage. iii) Finally rolling friction slows the rolling process until the nuclei reach a rigid configuration ("sticking" limit).

Depending on the inftial impact parameter, a collision will not necessar1ly go through all the different stages described above. Quasi-elastic collisions which are associated with large impact parameters proceed only through the first stage and only a small fraction of the initial angular momentum is imparted to the fragments. This fraction is proportional to the mass exchanged during collision. For smaller impact parameters in which 
20

Fig. 11. Fraction of excitation $\left(E^{*}\right)$ dissipated by $\gamma$-ray emission. 
Fraction of Excitation dissipated by $\gamma$-ray emission

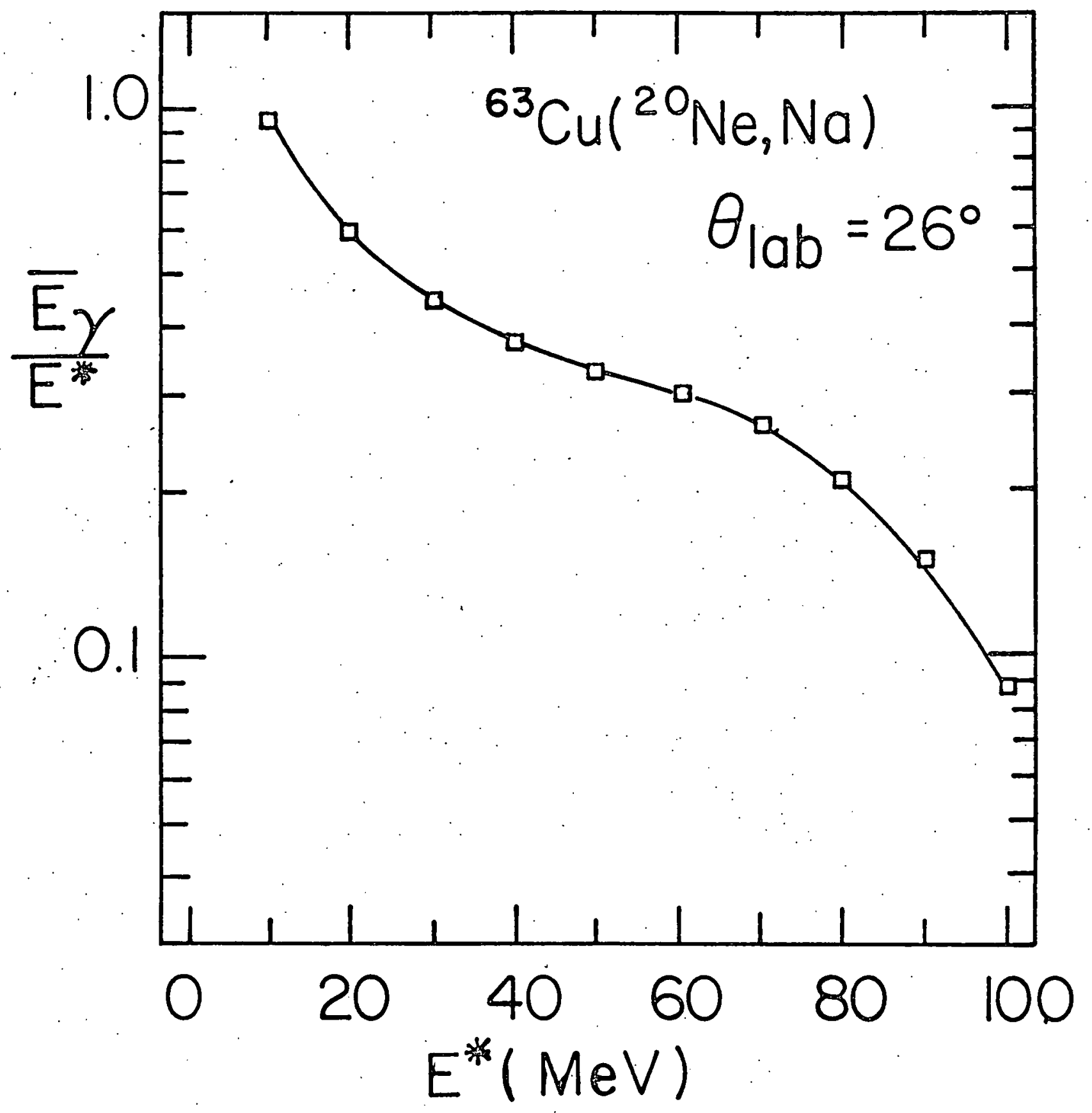

Fig. II 
the damping of energy is not complete; one can reach the second stage. These collisions where one observes a complete energy damping and mass equilibration are expected to reach the third stage.

For two rigid spheres undergoing tangential collision, the angular momentum transferred to the fragments increases from zero at the beginning of the sliding stage to $\frac{2}{7}$ of the initial angular momentum $\ell_{i}$ when the rolling stage is reached: The fraction $\frac{2}{7}$ is independent of the relative mass of the fragment. In the sticking stage, the angular momentum imparted to the fragments is given by

$$
I=\frac{J_{1}+J_{2}}{\mu R^{2}+J_{1}+J_{2}} \ell_{i}
$$

where $J_{1}$ and $J_{2}$ are the intrinsic moments of inertia of the fragments, $\mu$ their reduced mass and $R$ their separation at scission. For.fragments of equal mass $I=\frac{2}{7} \ell_{i}$ as in the rolling stage but the angular momentum transferred increased with the mass assymetry at the time of scission. Relation (3) can be rewritten as:

$$
I=\frac{\frac{2}{5}\left(A_{1}^{5 / 3}+A_{2}^{5 / 3}\right)}{\frac{A_{1} A_{2}}{A_{1}+A_{2}}\left(A_{1}^{1 / 3}+A_{2}^{1 / 3}\right)^{2}+\frac{2}{5}\left(A_{1}^{5 / 3}+A_{2}^{5 / 3}\right)} \ell_{i}
$$

In fig. 10, we have plotted the angular momentum transfer expected in the rolling and in the sticking case assuming the two limiting values of the initial angular momentum: $\ell=\ell_{c r}$ and $\ell=\ell_{g r}$ for projectile-like fragments. With $\mathrm{Z} \geq 12$ one cannot distinguish between rolling and sticking. For fragments with $\mathrm{Z}<12$, the energy spectra show a complete damping of the energy and one expects to have reached the sticking limit. However the experimental values of the angular momentum transfer lie below the theoretical 
limit for sticking and indicate an intermediate condition between rolling and sticking.

In figures 8 and 7 , the $\gamma$-ray multiplicities $\bar{M}_{\gamma}$ show a sharp drop for those fragments emerging with an energy smaller than the optimal energy for deep inelastic collision. These fragments emerging with a very low-energy are certainly associated to very elongated scission configuration and. according to relation (3), a large increase in the separation distance $\mathrm{R}$ leads to a decrease of the angular momentum transferred which manifests itself by a drop in the observed multiplicity.

\section{Width and Skewness of the Multiplicity Distribution}

Just as the average number of $\gamma$-rays reflects an average angular momentum, the distribution of the number of $\gamma$-rays about the average reflects a distribution in angular momenta. Knowledge of the distribution of angular momenta in the reaction products is very desirable. In the case of deep inelastic collision, the second and third moments of the $\gamma$-ray multiplicity have been measured in this work. For the ${ }^{20} \mathrm{Ne}$ and ${ }^{63} \mathrm{Cu}$ reaction the data are shown in Fig. 12. The moments of the $\gamma$-ray multiplicity are in each case evaluated at a single scattering angle and over a narrow energy window at the peak intensity of the deep inelastic yield for each atomic number. (In this portion of the spectrum, the contribution from reactions induced on carbon and oxygen contaminants in the target is small). One would expect that the width of the multiplicity distribution would be less in the case of the deep inelastic collision; the argument is as follows. The evaporation residues contain angular momenta from 0 to $\ell_{\text {crit }}$ and, for a $[2 \ell+1]$ triangular distribution, we would expect $\frac{\langle M\rangle}{\langle\sigma\rangle} \simeq \sqrt{8} \simeq 2.8$. We observe a value of 2.3 and attribute the discrepancy to additional broadening arising from particle 
Fig. 12. The multiplicity $\bar{M}_{\gamma}$, width $\sigma$, and skewness measured for the deep inelastic scattering of ${ }^{63} \mathrm{Cu}$ and ${ }^{20} \mathrm{Ne}$. The straight lines are to guide the eye. 


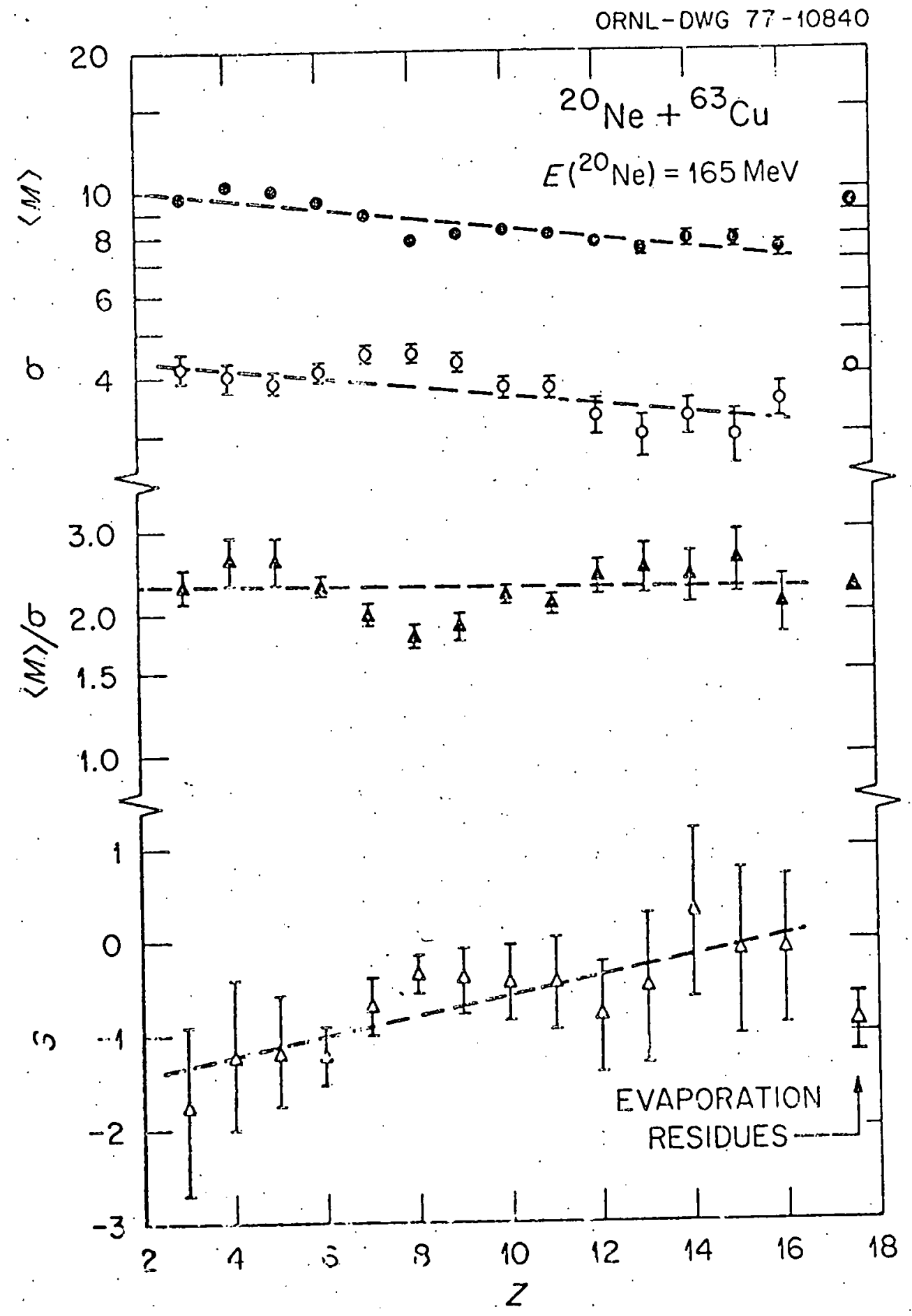

Fig. 12 
emission and statistical cascades. In the case of deep inelastic scattering we would expect a narrower distribution of angular momenta varying from $f \cdot l_{\text {crit }}$ to $f \cdot l_{g r}$ where $f$ represents the fraction of angular momentum transferred. Indeed, this should represent an upper limit on the width since we have selected a narrow angular and energy region of deep inelastic products and, if anything, this would narrow the range of impact parameter. Neglecting broadening from particle emission and from other effects such as statistical cascades, a variance $\sigma^{2} \cong 0.6$ would be expected for the multiplicity distribution. Assuming the broadening of the distribution by the effects mentioned above can be derived from the value deduced from the evaporation residues, a variance of $\sigma^{2} \simeq 7.6$ is predicted. Yet this value is only about one half of the measured variance. For example, $\sigma_{\text {experimental }}^{2}=15$ for deep inelastic products with $\mathrm{z}=5$. This implies an additional broadening of the transferred angular momentum distribution. Possible explanations for this are as follows.

1. The distribution of initial angular momenta contribution to deep inelastic scattering is much broader than $\ell_{\text {crit }} \rightarrow_{\mathrm{gr}} \ell^{*}$ Even if this were the case, however, the selection of reaction products. at a given energy and scattering angle should correspond to a narrow population of initial angular momenta. This follows provided that the angular momentum transfer $J$ is proportional to the initial orbital angular momentum, $J=f \times \ell$.

2. The constant $f$ in the relation $J=f \times \ell_{i}$ is not a constant but has itself a distribution. This would imply statistical processes in the transfer of angular momentum, a proposition which does not seem unreasonable. 
3. There are additional angular momenta induced at scission (the bending and wriggling just discussed) which would certainly broaden the angular momentum distribution while not greatly affecting the average value. This possibility has been suggested by Perrin and Peter. 5

4. We have assumed that the excited fragments produced in the deep inelastic collision reach equilibrium and decay in the same manner as compound nuclei produced in fusion reactions. This assumption may not be valid.

${ }^{5}$ N. Perrin and J. Peter, Preprint IPNO-RC-77-02, presented at the Winter School on Nuclear Physics, Zakopane (Poland) 2/77. 
(b) Energy Dissipation by Charged Particle Emission in Deep Inelastic Scattering

R. A. Dayras, ${ }^{*}$ J. H. Barker, ${ }^{* *}$ C. B. Fulmer, ${ }^{*}$ K. Geoffroy, ${ }^{*}$ M. Halbert, ${ }^{*}$ D. Hensley, ${ }^{*}$ D. G. Sarantites, D. Shapira, , R. Stokstad, A. Sne11, and K. Wikstrom

As it was shown in the previous section, the major portion of energy dissipation in the DI processes from the reaction ${ }^{63} \mathrm{Cu}\left({ }^{20} \mathrm{Ne}, \mathrm{Na}\right)$ at $165 \mathrm{MeV}$ goes in other than $\gamma$-ray energy. In order to obtain information on the number, type, energy and angular distribution of light charged particles emitted in the quasi-elastic- deep inelastic and fusion portions of the ${ }^{20} \mathrm{Ne}+{ }^{63} \mathrm{Cu}$ reaction at $165 \mathrm{MeV}$, we carried out an appropriate multi-coincidence experiment. A new $49.5 \mathrm{~cm}$ diameter scattering chamber with an oil "free" vacuum system specifically constructed for this experiment was employed. A continuously movable target was used and this resulted in no noticeable contaminant build-up.

The projectile-like fragments were detected in two gaseous-ionization $\triangle E x E$ heavy-ion telescopes positioned on each side of the beam at $25^{\circ}$ and $35^{\circ}$. Six 1ight particle $\Delta \mathrm{ExE}$ telescopes consisting of $35-65 \mu \mathrm{m} \Delta \mathrm{E}$ and $1500 \mu \mathrm{m}$ E Si surface barrier detectors were employed to record simultaneously all possible coincldences. Measurements were taken both in and out of the reaction plane defined by the beam and either of the heavy-ion telescopes.

All coincidence events were tagged with $\gamma$-ray multiplicity information derived from the number of NaI detectors that fired in coincidence. A

\footnotetext{
*Oak Ridge National Laboratory, Physics Division.

${ }^{* *}$ St. Louis University, Physics Department.
} 
total of eight $2^{\prime \prime} \times 3^{\prime \prime} \mathrm{NaI}$ detectors were employed in this experiment. The data from this very complex experiment are currently being analyzed. From this analysis a more complete picture of the overall energy balance for strongly damped collisions will be forthcoming. 


\subsection{NUCLEAR STRUCTURE AT HIGH ANGULAR MOMENTUM}

(a) Identification of a $\mathrm{J} \simeq 32$ Yrast Trap in ${ }^{148} \mathrm{~Tb}$

J. R. Beene, J. H. Barker, K. Geoffroy, J. Jastrzebski, M. L. Halbert, D. C. Hensley, D. G. Sarantites, and L. Westerberg.

As it was proposed last year a series of experiments were carried out for the purpose of identifying and characterizing the decay properties of some of the high-spin isomers that were reported by Pedersen et $\underline{\text { al. }}{ }^{6}$ in order to see whether these isomers are indeed describable as "yrast-traps" as originally described by Bohr and Mottelson ${ }^{7}$ and further discussed by others. 8

The first completed work was submitted for Publication in Phys. Rev. Letters. The abstract from that letter follows and a preprint is appended. (See Report No. COO-4052-14)

\section{ABSTRACT}

Delayed high multiplicity gamma radiation has been observed in reactions of ${ }^{16} 0$ with ${ }^{142} \mathrm{Nd}$. A group of 13 to 16 lines has been shown to originate from a single isomer in ${ }^{148} 8_{\mathrm{Tb}}$ with $\mathrm{T}_{1 / 2}=19 \pm 3 \mathrm{~ns}$, excitation energy $\sim 8.2 \mathrm{MeV}$ and spin in the range 28 to 34 .

${ }^{6} \mathrm{~J}$. Pedersen et al., Phys. Rev. Lett. $\underline{39}, 990$ (1977). ${ }^{7}$ A. Bohr and B.R. Mottelson, Phys. Scr. 10A, 13 (1974). $8_{K}$. Neergaard et al., Nucl. Phys. A262, 61 (1976); G. Anderson et al., Nuc1 Phys. A268, 2051 (1976); T. Dossing et a1., Phys. Rev. Lett. $\underline{39}$, 1395 (1976). 
(b) Discrete High-spin States in the Yrast Sequence of ${ }^{164} \mathrm{Yb}$

N. R. Johnson,,$^{*}$ I. Y. Lee, ${ }^{* *}$ T. T. Sugihara, ${ }^{* *}$ L. L. Riedinger, ${ }^{+}$C. R. Bingham, $^{+}$S. W. Yates, ${ }^{+}$and D. G. Sarantites

In order to determine the properties of very high-spin discrete states in ${ }^{164} \mathrm{Yb}$ we have carried out the reaction ${ }^{150} \mathrm{Nd}\left({ }^{20} \mathrm{Ne}, 6 \mathrm{n}\right){ }^{164} \mathrm{Yb}$ at ORIC. To enhance the desired reaction channel we employed a multiplicity filter consisting of an array of $14 \mathrm{NaI}$ detectors in all possible coincidences with three Ge(Li) detectors. For this purpose a second multiplicity lead shield was constructed at Washington University to house seven more 2 " $\mathrm{x} 3^{\prime \prime} \mathrm{NaI}$ detectors and new stand was constructed to permit positioning of the NaI detectors above and below the scattering chamber plane. To reduce the Doppler broadening the $\mathrm{Ge}(\mathrm{Li})$ detectors were located near $180^{\circ}$ and $0^{\circ}$ and thin self-supporting targets $\left(<1 \mathrm{mg} / \mathrm{cm}^{2}\right)$ were used to permit the excited recoiling nuclei to decay while in flight in vacuum. Analysis of the data showed the known states with $\mathrm{J}$ up to $22^{+}$. Three additional transitions were placed as the next three states on the basis of their coincidence and intensity relationships in the yrast sequence as seen in Fig. 13.

\footnotetext{
* Oak Ridge National Laboratory, Chemistry Division, 
Fig. 13. Backbending plot for ${ }^{164} \mathrm{Yb}$. showing the variation of the moment of inertia with spin or transition energy. 


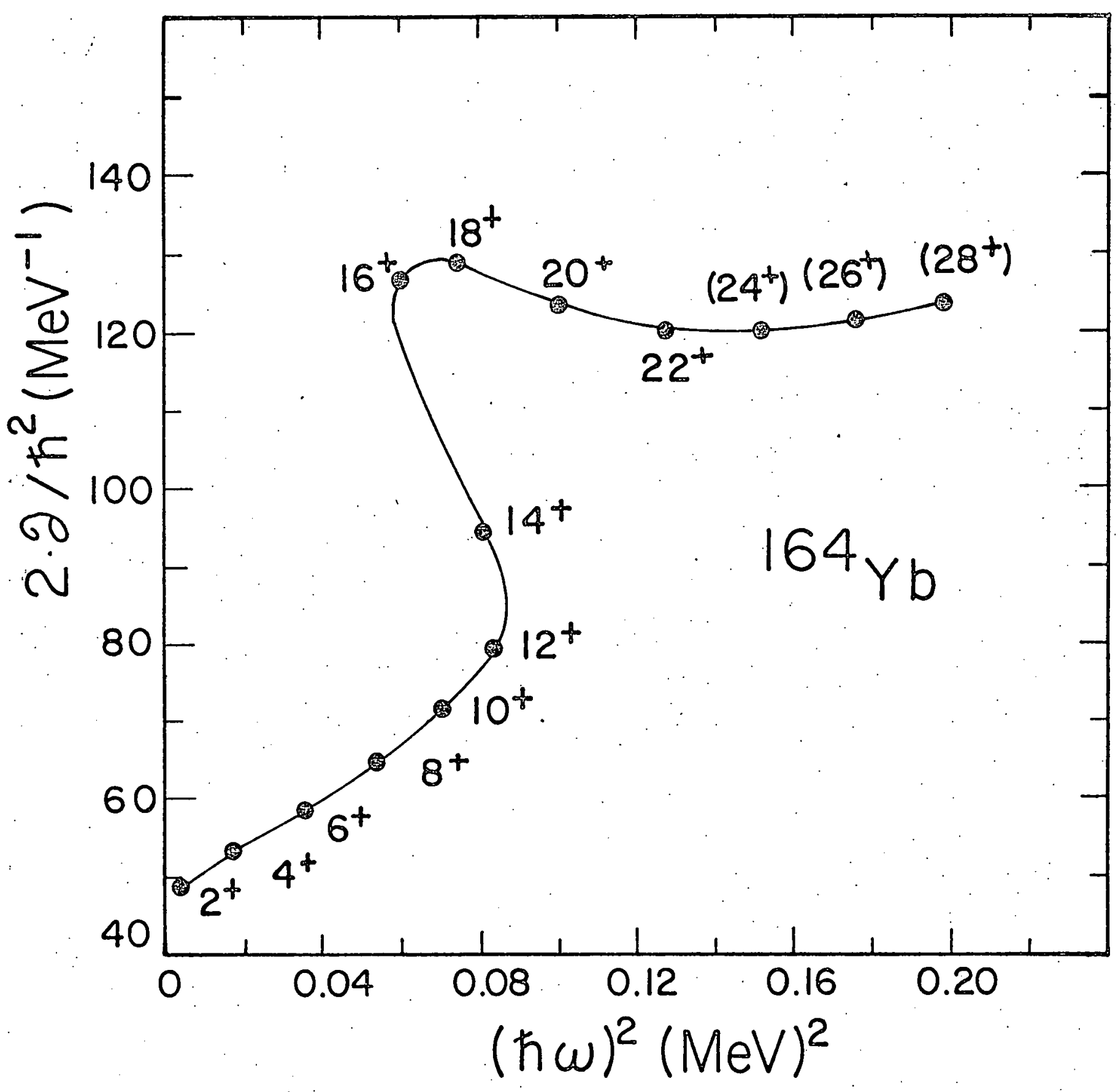

Fig.13 
(c) High-spin states in ${ }^{130} \mathrm{Ce}$

N. R. Johnson, ${ }^{*}$ I. Y. Lee, ${ }^{* *}$ L. L. Riedinger, ${ }^{+}$D. Sarantites, C. Bingham, ${ }^{+}$D. C. Hensley, ${ }^{* *}$ M. W. Guidry, ${ }^{+}$S. W. Yates, ${ }^{+}$R. L. Robinson $^{* *}$ and H. K. Carter ${ }^{+H}$

In a continued effort to excite and investigate nuclei in discrete states at very high angular momentum with the beams available at the ORIC. we selected the ${ }^{94} \mathrm{Zr}\left({ }^{40} \mathrm{Ar}, 4 \mathrm{n}\right){ }^{130} \mathrm{Ce}$ at $160 \mathrm{MeV}$ of incident energy. This choice was prompted on the basis of anticipated more favorable feeding pattern for reaching the yrast states at high $\mathrm{J}$.

To reduce Doppler broadening as in the previous experiment $1-3(\mathrm{~b})$ a very thin self-supporting target of ${ }^{94} \mathrm{Zr}\left(<1 \mathrm{mg} / \mathrm{cm}^{2}\right)$ was used to permit decay of recoiling nuclei in flight in the vacuum. To further reduce the Doppler broadening and the structure less background found in all spectra in similar investigations we have employed a $\mathrm{Ge}(\mathrm{Li})-\mathrm{NaI}$ anti-Compton spectrometer positioned at $0^{\circ}$ to the beam. In order to maintain reasonable detection efficiency the Washington University anti-Compton spectrometer ${ }^{9}$ consisting of a $6 \% \mathrm{Ge}(\mathrm{Li})$ detector and a $19 \mathrm{~cm}$ diameter by 14 $\mathrm{cm}$ long. NaI annulus was employed. This spectrometer can be placed so

\footnotetext{
*Oak Ridge National Laboratory, Chemistry Division.

** Oak Ridge National Laboratory, Physics Div.sion.

${ }^{* * *}$ Texas A\&M University and Oak Ridge National Laboratory, Chemistry Division.

$t_{\text {University of Tennessee, Department of Physics }}$

+University of Kentucky, Department of Chemistry

+ Oak Ridge National Laboratory; UNISOR

${ }^{9}$ L. Westerberg et al., Nucl. Inst. Meth. 145, 295 (1977).
} 
that the $\mathrm{Ge}(\mathrm{Li})$ detector is at $12 \mathrm{~cm}^{2}$. from the target and still obtain a suppression of the Compton events by a factor of $\sim 3$.

In order to enhance the desired channel a multiplicity filter consisting of $\operatorname{six} 2^{\prime \prime} \times 3^{\prime \prime}$ detectors was employed. To accomplish this and retain the proximity of the anti-Compton spectrometer to the target the lead shield "URCHIN" of Ref. was modified by cutting sufficient amounts of $\mathrm{Pb}$ to remove one of the peripheral detectors:

In this experiment four additional Ge(Li) detectors positioned pairwise at $30^{\circ}$ and $150^{\circ}$ to the beam (see Fig. 14) were used. Concidences between and of the four $\mathrm{Ge}(\mathrm{Li})$ and the anti-Compton spectrometer under multiplicity selection were recorded. In addition $\mathrm{Ge}(\mathrm{Li})-\mathrm{Ge}(\mathrm{Li})-\mathrm{mult}$ plicity events between the two $150^{\circ}$ detectors were recorded.

The data were aquired with the Tennecomp computer system employing a new. "multiplicity box" (a 4 x 16 overlap coincidence matrix) constructed at the ORIC. A special minature scattering chamber was also designed and fabricated at the ORIC.

Preliminary examiation of the data indicates a successful experiment. The full analysis of the data is currently in progress. 
Fig. 14. Two photographic views of the multi-coincidence apparatus for the high-spin experiment. A shows the lead shield for the anti-Compton spectrometer at $0^{\circ}$ to the beam direction, B shows the beam line and $\mathrm{C}$ one of the four $\mathrm{Ge}(\mathrm{Li})$ detectors. The six 2" $x 3^{\prime \prime}$ NaI detectors lie below the reaction plane. 

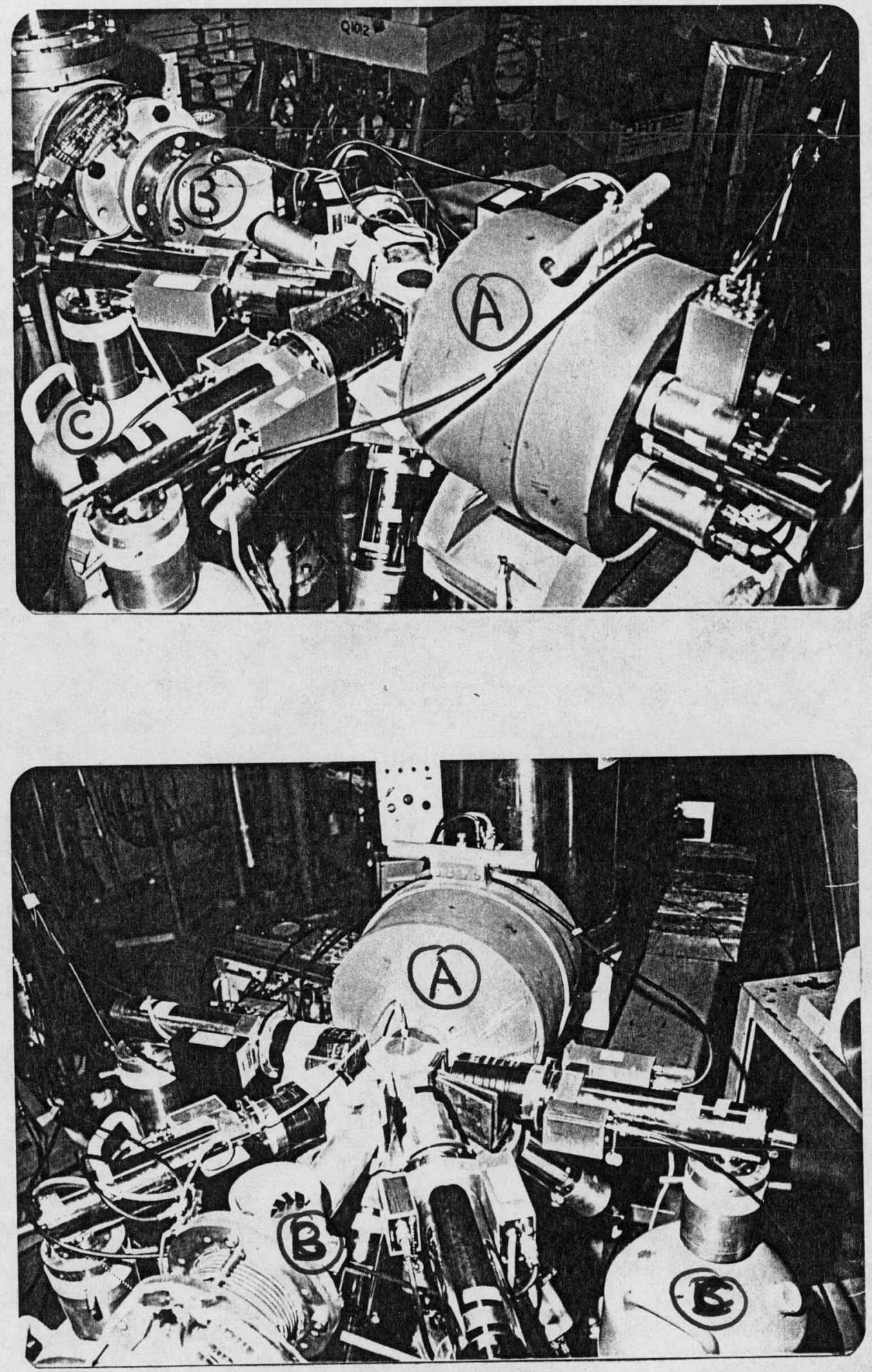

Fig. 14 
PHYSICAL REVIEW C

VOLUME I I, NUMBER 4; 1325

A PRIL 1977

(d) Lifetimes of ground-band states in ${ }^{192} \mathrm{Pt}$ and ${ }^{194} \mathrm{Pt}$

Noah R. Johnson, P. P. Hubert, ${ }^{*}$ and E. Eichler ${ }^{\dagger}$

Oak Ridge National Laboratory, 'Oak Ridge. Tennessee 37830

D. G. Sarantites and J. Urbon ${ }^{\S}$

Department of Chemistry. Washington University. St. Louis, Missouri 63130

S. W. Yates ${ }^{\sharp}$

- Department of Chemistry, University of Kentucky, Lexington, Kentucky 40506

Thomas Lindblad

Research Institute for Physics, S-10405 Stockholm 50, Sweden

(Received 18 October 1976)

The lifetimes of members of the ground-state bands in ${ }^{192} \mathrm{Pt}$ and ${ }^{194} \mathrm{Pt}$ have been measured by the Doppler-shift recoil-distance technique. These states were Coulomb excited by $149 \mathrm{MeV}{ }^{40} \mathrm{Ar}$ ions and measurements were carried out in coincidence with backscattered projectiles. The half-lives of the $2^{+}, 4^{+}$, and $6^{+}$states in ${ }^{192} \mathrm{Pt}$ and of the $2^{+}$and $4^{+}$, states in ${ }^{194} \mathrm{Pt}$ are $48.5 \pm 2.5,4.2 \pm 0.2,1.8 \pm 0.7,45.0 \pm 2.4$, and $3.7 \pm 0.2 \mathrm{ps}$, respectively.

NUCLEAR REACTIONS ${ }^{192.194} \mathrm{Pt}\left({ }^{40} \mathrm{Ar},{ }^{40} \mathrm{Ar}^{\prime} \gamma\right), E=149 \mathrm{MeV}$; measured lifetimes of

$2^{+}, 4^{+}$, and $6^{+}$states in ${ }^{192} \mathrm{Pt}$ and of $2^{+}$and $4^{+}$states in ${ }^{24} \mathrm{Pt}$; deduced $B(E 2)$ values, compared with theory.

Preprints of this article were submitted last year (C00-4052-10) 
(e) Structure of the weakly deformed 97,99 Ru nuclei

$$
\text { H.-C. Hseuh, }{ }^{+} \text {E. S. Macias and D. G. Sarantites }
$$

Department of Chemistry, Washington University

$$
\text { St. Louis; MO } 63130
$$

M. Brenner

Department of Physics, Abo Akademi, Abo, Finland

\section{W. Klamra}

Research Institute for Physics, Stockholm Sweden

\section{ABSTRACT}

The level structure and decay properties of levels in ${ }^{97} \mathrm{Ru}$ with $\mathrm{J}^{\pi}$ up to $27 / 2^{+}$and $4264.7 \mathrm{keV}$ of excitation; and in ${ }^{99} \mathrm{Ru}$ with $J^{\pi}$ up to $27 / 2^{-}$and $4223.0 \mathrm{keV}$ of excitation have been investigated via measurement of the energy and intensity of the prompt $\gamma$ rays and via $\gamma-\gamma$ coincidence and $\gamma-r a y$ multiplicity measurements following the ${ }^{95,97} \mathrm{Mo}(\alpha, 2 \mathrm{n}){ }^{97,99} \mathrm{Ru}^{*}(\gamma)$ reactions between 17 and $30 \mathrm{MeV}$. Level schemes for ${ }^{97} \mathrm{Ru}$ and ${ }^{99} \mathrm{Ru}$ were constructed. Only 9 of the proposed 25 levels in ${ }^{97} \mathrm{Ru}$ and 12 of 24 levels in ${ }^{99} \mathrm{Ru}$ were previously reported. $J^{\pi}$ assignments are proposed for levels in ${ }^{97} \mathrm{Ru}$ and ${ }^{99} \mathrm{Ru}$ based on $\gamma$-ray angular distribution and excitation function measurements. Even-parity bands decaying by $E 2$ cascades to the $5 / 2^{+}$ground state were observed in both nuclei. E2 cascades to the $11 / 2^{-}$level in ${ }^{99} \mathrm{Ru}$ and $7 / 2^{+}$level in ${ }^{97} \mathrm{Ru}$ were also observed. The level structure of ${ }^{97} \mathrm{Ru}$ was interpreted in terms of few particle configurations and was compared with the structures of ${ }^{95}$ Mo and. ${ }^{96,98}$ Ru. The level structure of ${ }^{99}$ Ru was 
compared with the results of a Coriolis coupling calculation. Good agreement in the level sequence of the yrast levels was obtained.

NUCLEAR REACTIONS: ${ }^{95} \mathrm{Mo}(\alpha, 2 \mathrm{n}){ }^{97} \mathrm{Ru}^{*}(\gamma),{ }^{97} \mathrm{Mo}(\alpha, 2 \mathrm{n}){ }^{99} \mathrm{Ru}^{*}(\gamma), \mathrm{E}_{\alpha}=17-30 \mathrm{MeV}$, enriched targets. Measured $E_{\gamma}, I_{\gamma}, I_{\gamma}(\theta), \gamma-\gamma$ coinc., $\gamma$-ray multiplicities, deduced ${ }^{97} \mathrm{Ru}$ and ${ }^{99} \mathrm{Ru}$ levels, $\mathrm{J}, \pi$, branching fractions; $\delta(\mathrm{E} 2 / \mathrm{MI})$; $\mathrm{Ge}$ (Li) detectors, Ge(Li)-NaI(T1) anti-Compton spectrometer, NaI(T1) detectors.

- This article was submitted for publication in The Physical Review $C$ and a preprint is appended (Report No. COO-4052-15). 


\section{PART 2. PRESENT STATUS OF INSTRUMENTATION AND ANALYSIS}

\subsection{APPARATUS FOR $\gamma$-RAY AND PARTICLE MULTIPLICITY EXPERIMENTS}

A large variety of apparatus for both $\gamma$-ray and charged particle or neutron multiplicity experiments were constructed and tested at Washington University. The majority of these are currently used and are available for other users of the ORIC facility. These are described briefly below.

\section{(a) Two anti-Compton Spectrometers}

The anti-Compton spectrometer that has been in use at Washington University over the last nine years ${ }^{10}$ has been employed extensivly in multiplicity experiments at the ORIC. Several early applications have been described in Ref. 9. A more recent application was described in Sect. 1.3 (b) of this report (See Fig. 14)

A second anti-Compton spectrometer employing a larger NaI annulus (10" diameter by 10" long) has been designed and the crystal is currently being fabricated. This instrument is to be made available at the HHIRF in Oak Ridge. The supporting gear and lead shield are being fabricated at the Washington University Chemistry Department machine shop. It is anticipated that a suppression factor of $8-10$ will be achieved with this instrument. It will be possible to position the spectrometer at angles covering the range $0^{\circ}-125^{\circ}$ to the beam direction.

\section{(b) The $\gamma$-ray Multiplicity "URCHIN" Lead Shields}

Two identical lead shields were constructed. Each of them houses seven 2 " diameter by 3 " long NaI detectors with sufficient shielding between neighboring detectors to render the crystal-to-crystal scattering

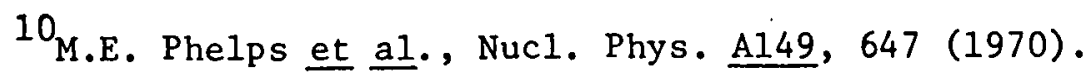


negligible. These two shields can be used either separately or together allowing up to 14 detectors to be used out of the horizontal reaction plane at a distance of $10 \mathrm{~cm}$ from the target.

- One of these shields has been used in the multiplicity measurments for Deep Inelastic. Scattering either with a $30-\mathrm{cm}$ or a $50-\mathrm{cm}$ diameter scattering chamber.

The two shields were used simultaneously (14 detectors) in conjunction with the $30-\mathrm{cm}$ chamber in the study of "yrast trap" isomers and in the study of very high-spin discrete yrast transitions.

Several experiments were also carried out where one shield was employed in conjunction with the magnetlc lens electron. spectrometer (see Sect: 2.2).

(c) Two 30-cm Diameter Multi-coincidence Scattering Chambers

These two chambers were constructed to provide maximum flexibility for $\gamma$-ray multiplicity work in conjunction with spectroscopic and/or reaction mechanism studies. Each of these includes several parts that can accommodate solid state detectors, several $2^{\prime \prime} \times 3^{\prime \prime}$ NaI detectors (10 $\mathrm{cm}$ from the target) or several $\mathrm{Ge}(\mathrm{L} i)$ detectors ( $8 \mathrm{~cm}$ from the target). The two hemispheres are available for either two multiplicity arrays ( $7 \mathrm{NaI}$ detectors each) or one multiplicity array and an anti-Compton spectrometer. A more detailed description of these chambers has been presented in Ref. 9.

(d) A 50-cm Diameter Multipole-coincidence Scattering Chamber

This scattering chamber was designed for maximum flexibility with multiple-coincidence arrangements involving NaI detectors, many light particle $\triangle E x E$ telescopes and two HI $\triangle E x E$ telescopes.' Provision exists for 
mounting the light particle telescopes in or out of the reaction plane. defined by the HI telescopes. Two arms can be moved from outside controls for changing detector angles without breaking the vacuum. A target lock has been constructed to permit changing targets and to continuously change target position without breaking the vacuum. A pressure of $1.2 \cdot 10^{-6}$ torr can easily be maintained in this oil free chamer.

\section{(e) A Multip1icity Routing Box}

A unit allowing the multiplicity of up to 16 detectors to be recorded by the fast overlap coincidence technique has been constructed and used routinely for experiments at the ORIC. A description is given in Ref. 9.

\subsection{A MAGNETIC LENS-Si(Li) VERSATILE CONVERSION ELECTRON SPECTROMETER}

A lens spectrometer modified ${ }^{11}$ for integral on-line conversion electrol spectrometry was bought from the University of Uppsala, Sweden, installed and tested at the Washington University cyclotron and then transported to the ORIC where it has been used in a variety of experiments employing heavy-ion induced reactions.

A manuscript describing in detail the performance of this instrument is being presently prepared for publication in Nucl. Inst. and Methods. A brief description is given here.

A cross-sectional view is shown in Fig. 15 where additional antiscattering baffles have been introduced compared to the spectrometer described in Ref. 11. The Si(Li) detector (A in Fig. 15) is mounted on a movable cold finger $\mathrm{B}$ that can remove the detector beyond a gate $\mathrm{C}$ so

11. Westerberg et a1. Nucl. Instru. Meth. 128, 61 (1975). 
$\dot{2}$

Fig. 15. A cross sectional view of the electron lens Si(Li) spectrometer. A is the $\mathrm{Si}(\mathrm{L} i)$ detector, B a gate valve, C the cold trap, D the lead plug, and $\mathrm{E}$ the anti-positron baffle. 
so that the lens and the scattering chamber can be let to air without warming up the detector. The cold-finger side of the instrument is shown in Fig. 16. The cold trap $D$ and the position $E$ of the anti-positron baffle are seen in Fig. 15. A special scattering chamber is shown. The latter is equipped with a vacuum lock to permit target or source changes without breaking the vacuum.

The lens is operated either at constant current ( $18 \%$ momentum acceptance, $0.7 \%$ absolute peak-efficiency at ${ }^{137} \mathrm{Cs}$ ) or in a sweeping current mode using a triangular wave generator at a period of $50 \mathrm{sec}(0.047 \%$ peak-efficiency at ${ }^{137}$ Cs for a broad sweep shown in Fig. 17). The increasing part of the efficiency curve follows the $\mathrm{B} \rho$ curve. The shape of this curve is generated by a computer code and has been carefully tested with absolute standards.

Usually the lens is operated in the sweeping mode. In this case both the energy and momentum for each event are recorded. This permits a considerable reduction of the events of partial energy deposition in the Si(Li) detector from high energy electrons.

The electron lens at the present time can be operated at $55^{\circ}$ or $125^{\circ}$ with respect to the beam direction and it has been used both in singles and in multi-detector coincidence arrangements. These applications are briefly described below.

(a) Conversion Coefficients from In-beam HI-induced Reactions

The sharp cut-off of the magnetic lens for low energy electrons makes this instrument particularly useful for conversion electron measurements. Thus electrons with energy down to $70 \mathrm{keV}$ can be detected without difficulties from $\delta$-rays. The quality of spectra obtained using a high 
Fig. 16. Detector side of the 1ens. A is the cold finger for the cold trap, B the detector cold finger, C the preamplifier and $\mathrm{D}$ the isolating gate valve. 


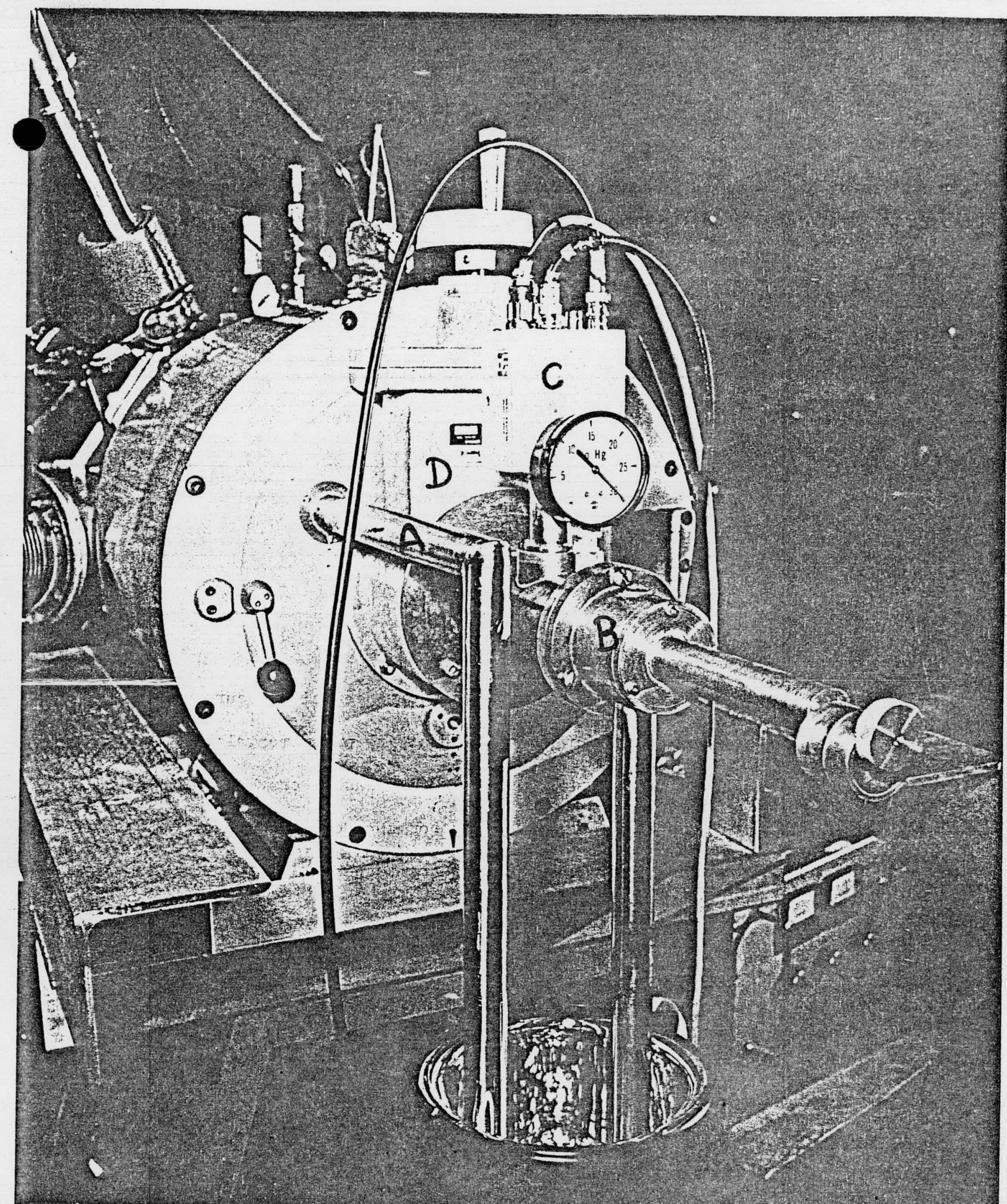




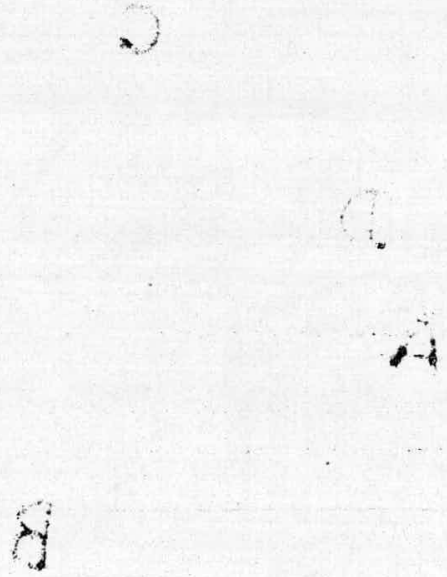

Fig. 17. Comparison of the theoretical relative efficiency curve (solid line) for the electron lens with experimental values from $e^{-}$sources (points). The dashed cruve is a theoretical curve that accounts for the somewhat longer time spent at the ends of the current sweep. 


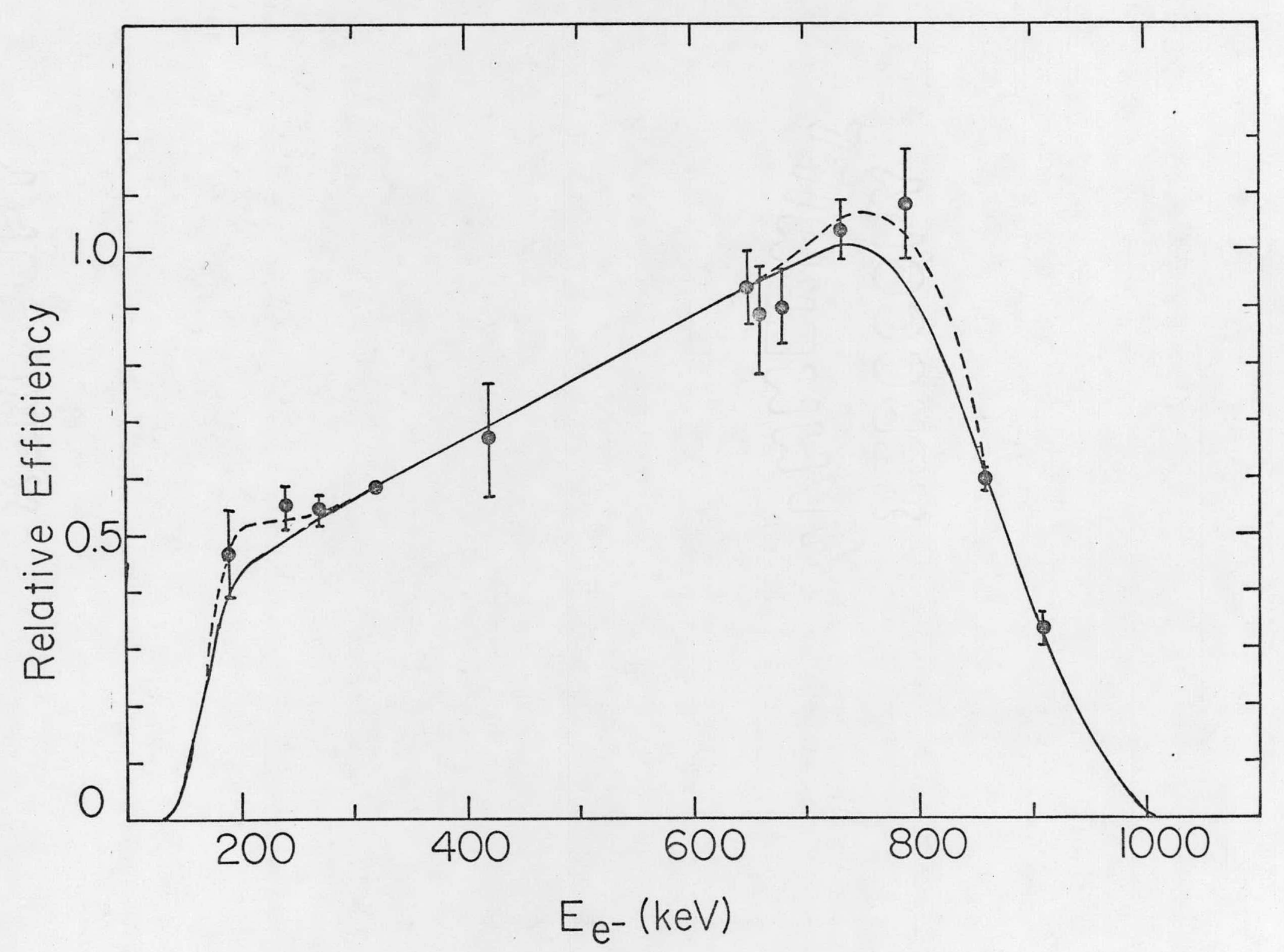

Fig. 17 
resolution $200 \mathrm{~m}^{2} \times 3 \mathrm{~mm}$ thick $\mathrm{Si}(\mathrm{Li}$ ) detector (with $1.8 \mathrm{keV}$ FWHM at 1000 $\mathrm{keV}$ ) is considerably better (peak to background ratio 3 times higher) than the $\gamma$-spectra with a typical $10 \% \mathrm{Ge}(\mathrm{Li})$ detector.

Improvements of the performance of the lens in terms of reducing Doppler broadening and target thickness effects are possible and will be incorporated in future experiments.

\section{(b) Conversion Electrons from the Continuum}

This particular instrument is we11 suited for studies of the conversion electrons from the underlying "continuum" radiation following heavy-ion induced reactions. An arrangement that was employed in such experiments (See Sect. 1.1-h) is shown in Fig. 18. The lens A is placed at $125^{\circ}$ to the beam. A seven $2^{\prime \prime} \times 3^{\prime \prime}$ NaI detector $\gamma$-multiplicity array is seen at $90^{\circ}$ to the beam. The lead shield supporting the NaI detectors is shown by B. An additional NaI detector is shown out of the plane. The multiplicity array was used to require a coincidence with conversion electrons for the purpose of reducing the extraneous background.

\section{(c) Multiplicity Experiments with Conversion Electrons}

It was possible to substitute the Ge(Li) detector with the lens spectrometer as the gating detector for $\gamma$-ray multiplicity measurements. The multiplicity of the $\gamma$-cascades coincident with discrete conversion electron lines could then be deduced.

A more important application, however, is the use of the lens as the gating detector for the purpose of measuring the $\gamma$-multiplicity as a function of transition energy of the continuum. This is to be contrasted with the use of NaI detectors for gating, where Compton contributions from higher energy transitions are interferring. 
Fig. 18. Photographic view of the lens in a multiplicity arrangement. A indicates the lens, B the lead shield for the multiplicity array and $\mathrm{C}$ is the Faraday cup. 


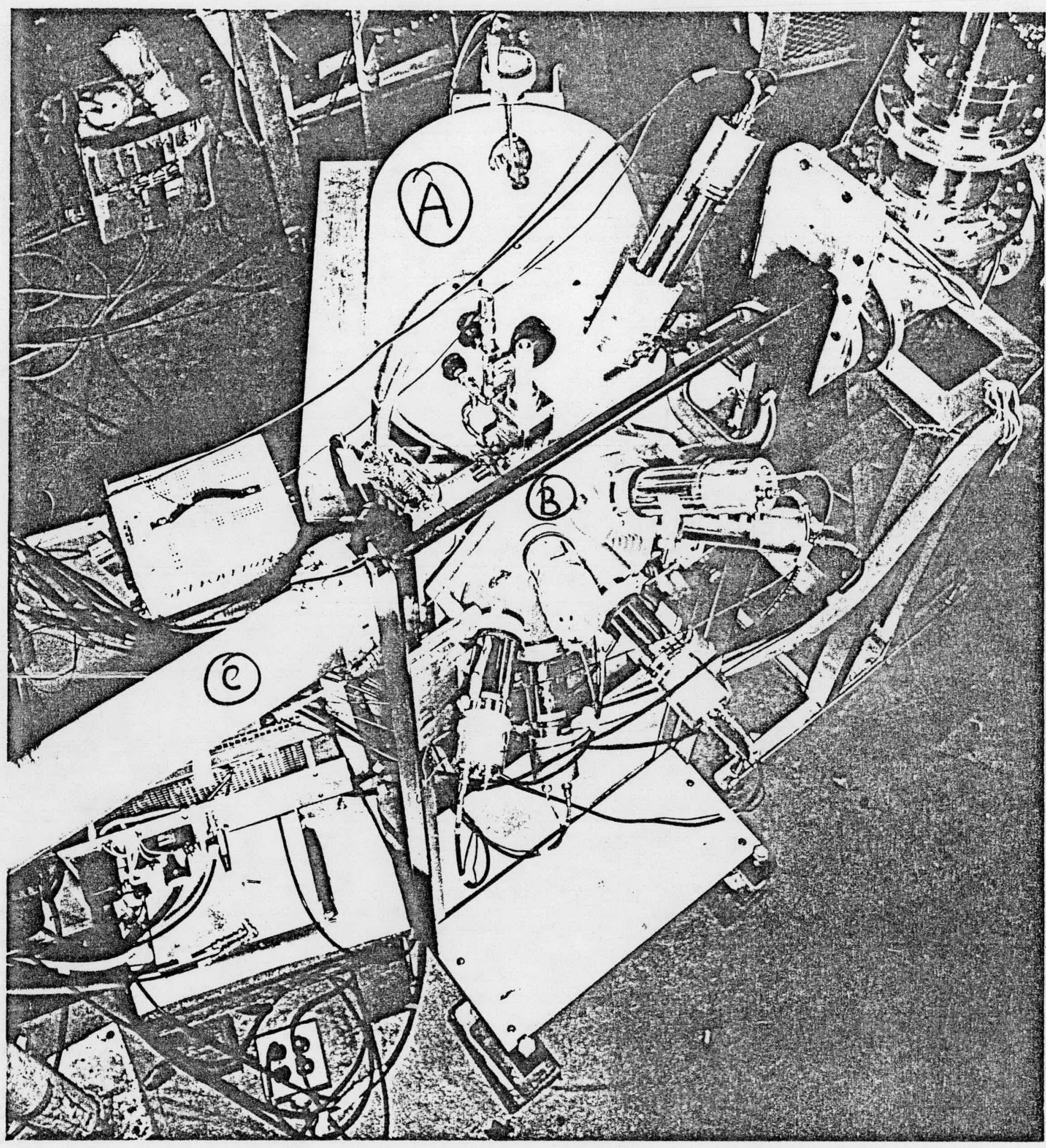

Fig. 18 
An arrangement that was used for such experiments and for conversion coefficient measurements from yrast trap isomers is shown in Fig. 19. The lens is indicated by $\mathrm{A}$, the dewar of a $\mathrm{Ge}(\mathrm{Li})$ detector by $\mathrm{B}$ and the cryopump by $C$. On top of the scattering chamber the target assembly is visible. Immediately below the scattering chamber a multiplicity assembly supporting $\operatorname{six} 2 " x 3^{\prime \prime}$ NaI detectors is located.

\subsection{A TOTAL ENERGY OR COINCIDENCE-SUMMING SPECTROMETER}

As it was proposed last year we proceeded with the design and construction of a coincidence-sum spectrometer usually called "total energy spectrometer." There are three major applications for this instrument. First, in the study of the effects of reaction mechanisms on the entry line. Second, in the use of the instrument in selecting the exit channel with the smallest number of emitted neutrons (at fixed bombardment energy) and therefore the highest angular momentum for spectrometric investigations at very high spin. The third is the application as a good anti-Compton spectrometer.

The instrument in the coincidence-sum application consists of (a) a 10 " diameter by $10^{\prime \prime}$ long NaI annulus with an axial hole of $2.5^{\prime \prime}$ diameter and a transverse $2.75^{\prime \prime}$ hole located at one end of the scintillator and (b) a second $7.5^{\prime \prime}$ diameter by 5.5" long NaI annulus with a $2.5^{\prime \prime}$ diameter axial hole. The two annular detectors will be used jointly to increase the sum efficiency.

The smaller annulus is already available and the larger one is on order and will be delivered early this coming fall. 
Fig. 19. Photographic view of the lens spectrometer in a multiplicity arrangement that permits two $\mathrm{Ge}(\mathrm{Li})$ detectors to be used in addition to the multiplicity array (shown below the scattering chamber). A is the lens, B the dewar for the Ge(Li) detector and $\mathrm{C}$ the upstream cryopump. 


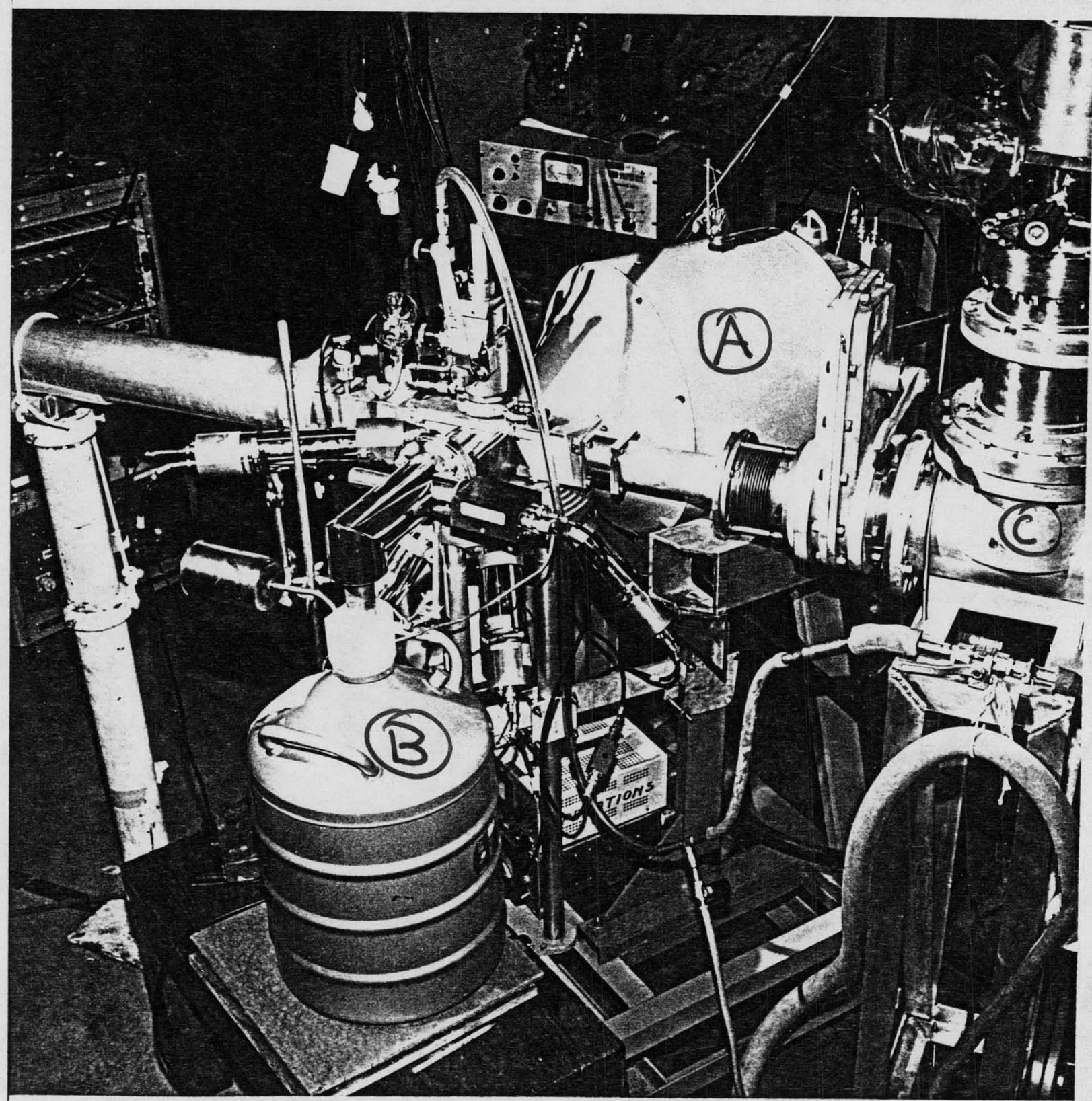

Fig.19 


\subsection{DETECTION EQUIPMENT}

\section{(a) $\mathrm{Ge}(\mathrm{Li})$ Detectors}

We currently have at Washington University two special $6 \% \mathrm{Ge}$ (Li) detectors suitable for use in either the anti-Compton or the coincidence sum application. A third $10 \%$ standard $\mathrm{Ge}(\mathrm{L} i)$ detector is also available.

A new special Ge(Li) compatible detector with the large $10^{\prime \prime} \times 10^{\prime \prime} \mathrm{NaI}$ annulus is also on order from ORNL funds.

\section{(b) Light-particle $\triangle$ ExE Telescopes}

At the present time we have four light-particle $\triangle E x E$ telescopes each consisting of a $65 \mu \mathrm{m}$ thick $150 \mathrm{~mm}^{2}$ transmission counter and a $1500 \mu \mathrm{m} 200$ $\mathrm{mm}^{2} \mathrm{E}$ counter. In addition ORIC has three additional telescopes equipped with thinner (down to $30 \mathrm{\mu m}$ ) telescopes. This set of seven telescopes has already been used simultaneously in several experiments.

\section{(c). HI Gas-proportional $\triangle E x E$ Telescopes}

Two gas-proportional $\triangle \mathrm{ExE}$ heavy-ion counters have been constructed and used in several coincidence experiments. These are mirror images of each other and incorporate a large area $200 \mathrm{~mm}^{2} \times 1500 \mu \mathrm{m}$ E counter ( $\sim 10 \mathrm{mstr}$ subtended solid angle).

\section{(d) Neutron Detectors}

We have designed and fabricated a series of detectors for use in timeof-flight experiments with the ORIC. These detectors have been designed for maximum coincidence efficiency and acceptable time resolution for experiments at the ORIC ( $\sim 3 \mathrm{~ns}$ time resolution in the beam microstructing). Four detectors consist of 12" diameter $3^{\prime \prime}$ long NE-102 plastic scintillators viewed by an RCA 4522 5" diameter photomultiplier tube. These are capable of providing large solid angle and a timing resolution of $2.5 \mathrm{~ns}$. 
Two smaller detectors $10 "$ diameter $2 "$ long have also been made and used. We also have a $5^{\prime \prime} \times 5^{\prime \prime} \mathrm{NE}-213$ scintillator capable of $\mathrm{n}-\gamma$ pulse shape discrimination and are fabricating several smaller ones ( $\left.5^{\prime \prime} \times 2^{\prime \prime} \mathrm{NE}-213\right)$. with these considerably better resolutions ( $<0.6 \mathrm{~ns}$ can be achieved).

\section{(e) Si(Li) Conversion Electron Detectors}

We have acquired a high resolution $\mathrm{Si}(\mathrm{Li})$ detector $(1.8 \mathrm{keV}$ FWHM at $1000 \mathrm{keV}$ and $800 \mathrm{eV}$ at $100 \mathrm{keV}$ ) and have used this detector effectively in conjunction with the electron lens for studies of conversion electrons from heavy-ion induced reactions.

\section{(f) Large NaI Detectors}

In addition to the fourteen $2 "$ x 3 " NaI detectors of the multiplicity array and the large annular detectors for the anti-Compton and coincidence sum spectrometer we have acquired a $5^{\prime \prime}$ diameter $4^{\prime \prime}$ long NaI detector for the purpose of studying the properties of the $\gamma$-ray continuum.

\subsection{DATA ACQUISITION AND REDUCTION}

(a) The ORIC SEL Computers as Data Acquisition Systems

The majority of the experiments that we have initiated at the ORIC were carried out using the SEL computers available there. Due to the particular needs for our experiments it was essentially always necessary to develop new software programs for data acquistion and for reduction for each new experiment. This task was carried out extremely well by D. Hensley who worked closely with us in all our experiments.

The data acquisition system in its present form includes eight high resolution stretchers (PACE system) and up to 18 bit of external digital information for word tagging. In addition a CAMAC based system is available 
that includes up to 16 additional lower resolution $\mathrm{ADC}$ 's and several eightfold logical coincidences latched for binary information. This system at the present time provides up to 24 parameters $(8+16)$ for analysis with considerable additional logical tagging by external conditions.

(b) Data Reduction at the ORIC Facility

As it was mentioned above (Sect. 2.5 a) the software for scanning the event tapes from all our experiments at the ORIC are now available. Complete documentation exists for most of these programs.

In addition a considerable number of data handling programs are now available on the SEL disk library for easy access and use. Some of these are (a) LIZA: a versatile data handling program with multiple display on a graphics scope and peak fitting capabilities and (b) SNAP: a versatile program for display and efficient analysis of 2 parameter data ( $\triangle E x E$ or $\Delta t \times E$, etc.):

In addition special programs developed for further calculations in this work have been adopted for the SEL computer for more efficient use. These include: (a) GETM a general $\gamma$-ray multiplicity program, and (b) UNFOLD a spectrum unfolding program capable of unfolding $\gamma$-ray spectra.

(c) Data Reduction at Washington University

The problems associated with data reduction at Washington University are discussed in Part II of the accompanying proposal. 
PART 3. SCIENTIFIC AND TECHNICAL PERSONNEL

The principal investigator has spent the summer of 1976 at ORIC under an ORAU faculty participation fellowship, and the summers of 1977 and 1978 as a consultant at ORIC.

other scientific personnel associated with this project include Professor J. H. Barker of St. Louis University, Dr. L. Westerberg of the University of Uppsala as research associate (February, 1976 - February 1978), Dr. Katherine Geoffroy as postdoctoral research associate (July, 1977 present) and Dr. K. Wikström of the Research Institute for Physics, Stockholm (February 1978 - present). Mr. R. Woodward has been a research assistant since September 1977. and Mr. B. J. Smith an undergraduate assistant during the summer of 1977 .

Major collaborators from ORIC are: Drs. M. L. Halbert, D. C. Hensley, R. G. Stokstad, R. A. Dayras, J. R. Beene, N. R. Johnson and I. Y. Lee. Collaborators from IUCF are Drs. J. Jastrzebski and P. P. Singh, and graduate research assistants M. Sadler, J. Wiggins and. H. Karwowski.

Technical staff involves design engineer, J. Hood, machinists G. Kretschmann, P. Shockely and W. J. Gray, cyclotron engineer R. Head and electronics engineer L. Djordjevic. 
PART 4. PUBLICATIONS

\subsection{Papers Published in 1976-1978}

1. "Transition rates of high-spin states in ${ }^{56} \mathrm{Co}$ and ${ }^{56} \mathrm{Fe}$. Possible coexistence of prolate- and oblate-like configurations in ${ }^{56} \mathrm{Fe} . "$

D.G. Sarantites, J. Urbon, L.L. Rutledge, Jr., Phys. Rev. 14C, 1412 (1976).

2. "Decay of 39 -day ${ }^{103} \mathrm{Ru}$ and 17 -day ${ }^{103} \mathrm{Pd}$ to the levels of ${ }^{103} \mathrm{Rh} . "$

E.S. Macias, M.E. Phelps, D.G. Sarantites, and R.A. Meyer, Phys. Rev. 14C, 639 (1976).

3. "Properties of ${ }^{93} \mathrm{Mo}$ via ${ }^{93} \mathrm{Nb}(\mathrm{p}, \mathrm{n}){ }^{93} \mathrm{Mo}^{*}(\gamma)$ reaction spectrometry."

L.L. Rutledge, Jr., E.S. Macias, and D.G. Sarantites, Phys. Rev. 13C, 2166 (1976).

4. "Coulomb excitation of ${ }^{99}$ Tc."

L.-G. Svensson, D.G. Sarantites and A. Bäcklin, Nuc1. Phys. A267, 190 (1976).

5. "Gamma-ray multiplicities in evaporation residues formed in bombardments of ${ }^{150} \mathrm{Nd}$ by ${ }^{20} \mathrm{Ne} . "$

D.G. Sarantites, J.H. Barker, M.L. Halbert, D.C. Hensley, R.A. Dayras,

E. Eichler, N.R. Johnson and S.A. Gronemeyer, Phys. Rev. 14C, 2138 (1976).

6. "Lifetimes of ground-band states in ${ }^{192} \mathrm{Pt}$ and ${ }^{194} \mathrm{Pt} . "$

N.R. Johnson, P.P. Hubert, E. Eichler, D.G. Sarantites, J. Urbon, S.W. Yates, and Th. Lindblad, Phys. Rev. 15C, 1325 (1977).

7. " $\gamma$-Ray multiplicities from auclear reactions: theory, instrumentation and analysis."

L. Westerberg, D.G. Sarantites, R. Lovett, J.T. Hood, J.H. Barker. C.M. Currie, and N. Mullani, Nucl. Instr. Meth. 145, 295 (1977).

8. "Reactions of ${ }^{20} \mathrm{Ne}$ on ${ }^{150} \mathrm{Nd}$ at $175 \mathrm{MeV}$." M.L. Halbert, R.A. Dayras, R.L. Ferguson, F. Plasil and D.G. Sarantites Phys. Rev. 17C, 155 (1978). 
9. "Angular-momentum effects in pre-equilibrium processes." D.G. Sarantites, L. Westerberg, R.A. Dayras, M.L. Halbert, D.C. Hensley and J.H. Barker, Phys. Rev. 17C, 601 (1978).

10. "Multiplicity of the $\gamma$-cascades in the ${ }^{61} \mathrm{Cu}$ continuum: dependence on excitation energy of the entry states."

J.H. Barker, D.G. Sarantites, 0̈. Skeppstedt, E. Wallander, G. Alenius, and S.-E. Arnel1, Phys. Rev. C (in. press).

11. "Multipolarities of the yrast and statistical cascades in ${ }^{150} \mathrm{Nd}\left({ }^{20} \mathrm{Ne}, \mathrm{xn} \gamma\right)$ reactions."

L. Westerberg, D.G. Sarantites, K. Geoffroy, R.A. Dayras, J.R. Beene, M.L. Halbert, D.C. Hensley, and J.H. Barker, Phys. Rev. Lett. (in press;

12. "Pre-equilibrium effects in fusion of ${ }^{12} \mathrm{C}$ and ${ }^{158} \mathrm{Gd} . "$ July 1978)

D.G. Sarantites, L. Westerberg, M.L. Halbert, R.A. Dayras, D.C. Henley, and J.H. Barker, Phys. Rev. C (in press for August, 1978).

13. "Pre-equilibrium particle emission from fusion of ${ }^{12} \mathrm{C}+{ }^{158} \mathrm{Gd}$ and $20 \mathrm{Ne}+{ }^{150} \mathrm{Nd} . "$

L. Westerberg, D.G. Sarantites, D.C. Hensley, R.A. Dayras, M.L. Halbert, and J.H. Barker, Phys. Rev. C (in press for August, 1978).

\subsection{Papers submitted for publication}

1. "A high-spin isomer in ${ }^{148} \mathrm{~Tb}$." J.R. Beene, M.L. Halbert, D.C. Hensley, D.G. Sarantites, L. Westerberg, K. Geoffroy, J.H. Barker, and J. Jastrzebski, Phys. Rev. Lett.

2. "Structure of the weakly deformed ${ }^{97} \mathrm{Ru}$ and ${ }^{99} \mathrm{Ru} . "$ R. Hseuh, E.S. Macias, D.G. Sarantites, W. Klamra and M. Brenner, Phys. Rev. C. 


\subsection{Presentations in meetings (1976-1978)}

1. "Investigations of the reactions of ${ }^{20} \mathrm{Ne}$ and ${ }^{150} \mathrm{Nd}$ at $175 \mathrm{MeV}$ via $\mathrm{\gamma}$-ray multiplicity measurements."

D.G. Sarantites, L. Westerberg, J.H. Barker, S.A. Gronemeyer, M.L. Halbert, R.A. Dayras, D.C. Hensley, E. Eichler, and N.R. Johnson. Invited paper, ACS meeting in San Francisco, September, 1976.

2. "Excitation energy dependence of the multiplicity of the $\gamma$ cascades following the ${ }^{58} \mathrm{Ni}\left(\alpha_{20}, \mathrm{p}\right){ }^{61} \mathrm{Cu}$ reaction."

J.H. Barker, D.G. Sarantites, 0. Skeppstedt, G. Alenius, and S.-E. Arnell, Bul1. Am. Phys. Soc. 21, 967 (1976) paper AD5

3. "High-spin states in 93 Mo."

L.L. Rutledge, Jr., E.S. Macias and D.G. Sarantites, Bull. Am. Phys. Soc. 21, 994 (1976).

4. "Gamma-ray multiplicites from reactions forming the compound nucleus ${ }^{100} \mathrm{Ru} . "$ L. Westerberg, D.G. Sarantites and E.S. Macias, Bul1. Am. Phys. Soc. 21, 995 (1976) paper DC8.

5. " $\gamma$-Ray multiplicity in DI reactions."

R.A. Dayras, R.G. Stokstad, M.L. Halbert, D.C. Hensley, A.H. Sne1l, R.L. Robinson, C.B. Fulmer; D.G. Sarantites and L. Westerberg, Bul1. Am. Phys. Soc. 22 , 593 (1977), paper GF 8 .

6. "Fusion cross sections for $175-\mathrm{MeV}{ }^{20} \mathrm{Ne}$ on ${ }^{150} \mathrm{Nd} . "$ M.L. Halbert, R.A. Dayras, F. Plasil, R.L. Ferguson and D.G. Sarantites, Bu11. Am. Phys. Soc. 22, 630 (1977) paper JE13.

7. "Decay of ${ }^{170} \mathrm{Yb}^{*}$ compound nuclel at $132 \mathrm{MeV}$ of excitation formed in reactions of ${ }^{20} \mathrm{Ne}$ with ${ }^{150} \mathrm{Nd}$ and ${ }^{12} \mathrm{C}$ with ${ }^{158} \mathrm{Gd} . "$

L. Westerberg, D.G. Sarantites, R.A. Goldworm, J.H. Barker, M.L. Halbert, R.A. Dayras, and D.C. Hensley, Bull. Am. Phys. Soc. 22, 630 (1977) paper JE14. 
8. " $\gamma$-Ray multiplicities from the decay of ${ }^{170} \mathrm{Yb}^{*}$ compound nuclei at excitation energies of 67-94 MeV formed in reactions of ${ }^{4} \mathrm{He}+{ }^{166} \mathrm{Er}$ and $20 \mathrm{Ne}+{ }^{150} \mathrm{Nd} . "$

D.G. Sarantites, L. Westerberg, J.H. Barker, R.A. Dayras, M.L. Halbert and D.C. Hensley, Bu11. Am. Phys. Soc. $22 ; 630$ (1977) paper JE15.

9. "Pre-equilibrium and equilibrium evaporation following fusion reactions with heavy-ions."

D.G. Sarantites, L. Westerberg, R. Goldworm, J.H. Barker, M.L. Halbert, R.A. Dayras, D.C. Hensley and N.R. Johnson, Invited talk ACS Symposium for E. Eichler, September 3, 1977.

i. . "Non-equilibrium effects in fusion of ${ }^{12} \mathrm{C}$ and ${ }^{158} \mathrm{Gd} . "$ M.L. Halbert, R.A. Dayras, D.C. Hensley, D.G. Sarantites, L. Westerberg, and J.H. Barker, Bull. Am. Phys. Soc. 23, 503 (1978) paper AG10.

11. "Pre-equilibrium neutron and alpha emission from fusion of ${ }^{12} \mathrm{C}+{ }^{158} \mathrm{Gd}$ to ${ }^{170}$ Yb at $132 \mathrm{MeV}$."

D.G. Sarantites, L. Westerberg, D.C. Hensley, R.A. Dayras, M.L. Halbert, and J.H. Barker, Bul1. Am. Phys. Soc. 23, 503 (1978) paper AG11.

12. "Non-equilibrium effects in reactions of ${ }^{16} 0$ with ${ }^{154} \mathrm{Sm} . "$

K.A. Geoffroy, D.G. Sarantites, L. Westerberg, J.H. Barker, R.A. Dayras, M.L. Halbert, and D.C. Hensley, Bull. Am. Phys. Soc. 23, 503 (1978) paper AG12.

13. "Multiplicity and Multipolarity of the $\gamma$-continuum for ${ }^{150} \mathrm{Nd}\left({ }^{20} \mathrm{Ne}, \mathrm{xn}\right)$ reactions at 115 and $130 \mathrm{MeV} . "$

L. Westerberg, D.G. Sarantites, K. Geoffroy, R.A. Dayras, J. Beene, M.L. Halbert, and D.C. Hensley, Bull. Am. Phys. Soc. 23, 504 (1978) paper AG15. 
14. "An investigation of high-spin isomeric states near the $N=82$ closed she11."

J.R. Beene, M.L. Halbert, D.C. Hensley, L. Westerberg, D.G. Sarantites, K. Geoffroy, and J. Jastrzebski, Bu11: Am. Phys. Soc., 23, 627 (1978) paper KF2.

15. "High-spin states in ${ }^{164} \mathrm{Yb} . "$ N.R. Johnson, I.Y. Lee, T.T. Sugihara, D.C. Hensley, J.R. Beene, L.L. Riedinger, C.R. Bingham, S.W. Yates, and D.G. Sarantites, Bull. Am. Phys. Soc. 23,627 (1978) paper KF8.

16. "Gamma-ray multiplicities in reaction products from $80 \mathrm{MeV}$ proton bombardment of ${ }^{62} \mathrm{Ni}$ and ${ }^{169} \mathrm{Tm} . "$ J.D. Wiggins, P.P. Singh, H. Karwowski, M.E. Sadler, J.' Jastrzebski, D.C. Sarantites, and L. Westerberg, Bull. Am. Phys. Soc. 23, 626 (1978) paper KE13.

17. "Multiplicities of gamma transitions observed in ${ }^{6}$ i induced reactions." H. Karwowskł, J.D. Wiggins, P.P. Singh, M.E. Sadler, J. Jastrzebski, D.G. Sarantites, and L. Westerberg, Bul1. Am. Phys. Soc. 23, 626 (1978) paper KE14. 